\title{
A Novel Zero-Watermarking Scheme Based on NSCT-SVD and Blockchain for Video Copyright
}

\author{
Xiangqi Wu \\ Hainan University \\ Peng Ma \\ Hainan University \\ Zihan Jin \\ Hainan University \\ Yuxuan Wu \\ Hainan University \\ Wei Ou ( $D$ ouwei@hainanu.edu.cn ) \\ Hainan University \\ Wenbao Han \\ Hainan University
}

\section{Research Article}

Keywords: Video copyright, Blockchain, Zero-watermarking, Privacy, Key-frames extraction

Posted Date: March 15th, 2021

DOl: https://doi.org/10.21203/rs.3.rs-288335/v1

License: (c) (1) This work is licensed under a Creative Commons Attribution 4.0 International License.

Read Full License 


\title{
A novel zero-watermarking scheme based on NSCT-SVD and blockchain for video copyright
}

\author{
Xiangqi $\mathrm{Wu}^{1}$, Peng $\mathrm{Ma}^{1}$, Zihan $\mathrm{Jin}^{1}$, Yuxuan $\mathrm{Wu}^{1}$, Wei $\mathrm{Ou}^{1^{*}}$ and Wenbao $\mathrm{Han}^{1}$ \\ ${ }^{1}$ School of Computer Science and Cyberspace Security, Hainan University, 570228, Haikou, \\ China
}

${ }^{*}$ Correspondence should be addressed to Wei Ou: ouwei@hainanu.edu.cn

\begin{abstract}
With the advent of information era, issues about video copyright continue to emerge. In the grey industrial chain of piracy, the film industry is a major disaster area so far. The current protection schemes for video copyright are inefficient and costly so that video industry still has to face the infringement. Research on a simple and efficient protection technology of video copyright is urgent. To address the inefficiency and irregularity of the current protection schemes for video copyright, this paper proposes a simple and efficient protection scheme for video copyright based on zerowatermarking and blockchain. The results show that the proposed key-frames extraction method of proposed scheme performs well and achieves low-cost protection of video copyright in pratical environment. In addition, video zero-watermarking algorithm of proposed scheme works well with blockchain. Proposed scheme improves some extent on the database centralization and evidential difficulties of traditional video zero-watermarking schemes, and it is more reliable, simpler and more effective than traditional video zero-watermarking schemes.
\end{abstract}

Keywords: Video copyright, Blockchain, Zero-watermarking, Privacy, Key-frames extraction

\section{Introduction}

Since the turn of the millennium, with the development of video products, the demand for video has risen quickly. However, some illegal application of video damage the owners' copyrights. Against this background, a reliable protection scheme for video copyright has become extremely necessary and urgent in 21 st century.

The development of technology for copyright protection is closely related to the demand for video copyright protection. With video springing up as the main way of information dissemination all over the world, there is a greater need for protection scheme of video copyright. In particular, TikTok, YouTube and Hollywood, the three organizations that represent the three most dominant approaches in video industry today (such as short-form video, self-published video and digital cinema). They all have a quite strong need for reliable protection scheme of video copyright. Hollywood loses 64.6 billion dollars for pirated videos every year. TikTok and YouTube have been even more so. At present, the research on video copyright protection is supported by a number of government agencies around the world, because of the application prospects of reliable protection scheme for video copyright, the urgency of copyright owners for their needs and the importance of economic development.

The reliable protection scheme for video copyright protects the economy. There was a time when a large number of companies went out of business around the world due to piracy and all sectors of society were once caught in a vicious cycle of "innovation - piracy - worse product lower revenue - company goes bankrupt - fewer jobs - lower wages - worse product". Piracy is a scourge that must be removed from individuals, societies, countries and even human civilization. It is fair to say that reliable protection scheme for video copyright protects far more than just the economy, but it protects the most precious part of 
human civilization which may be the cornerstone of human development. This is why a reliable protection scheme for video copyright is so necessary. Unfortunately, the current research priority is still on traditional image copyright authentication and less on video copyright authentication in the worldwide. The video copyright authentication still needs to be developed.

With the continuous research on information hiding technology, digital watermarking technology of information hiding technology is playing an increasingly important role in the copyright protection of digital works. The main problems with existing protection schemes of video copyright as follows:

- High complexity of watermarking algorithm: In real processes, the complexity of the algorithm is closely related to the actual operational efficiency. Excessive complexity will significantly reduce operational efficiency to the point where commercialization is impossible.

- Poor robustness: There is a high probability that the watermark will be corrupted after multiple transform in the current network architecture or after compression, rendering it ineffective for copyright protection purposes.

- Weak stealthy: The addition of watermark to some videos has a visually perceptible impact on the original quality of the video, making it less commercially viable.

- Too single-function: Most current schemes have single function on copyright authentication. However, in an actual scenario of copyright protection, there will be some functions such as traceability of rights. The deficiency of existing schemes makes the actual process of rights maintenance quite complicated.

To solve these pressing protection issues of video copyright, we design a video key-frames extraction algorithm based on distance threshold clustering and a colour zero-watermarking algorithm based on combination of NonSubsampled Contourlet Transform (NSCT) with Singular Value Decomposition (SVD) in proposed scheme. We innovatively combine video zero-watermarking scheme with blockchain to address problem of data management in resent protection scheme on video copyright [1].

The rest part of the paper is organized as follows. The related works are in Related works section. We introduce the development of digital copyright protection and the latest research on image blind watermarking, zero-watermarking and video watermarking. The proposed scheme is elaborated in Scheme section. Firstly, the overall architecture of system for video copyright protection is introduced, and how to combine zero-watermarking with blockchain to realize copyright protection function is explained. Secondly, we introduce the selection algorithm of the key-frames from video. Thirdly, we introduce a zero-watermarking algorithm for key-frames based on NSCT and SVD. Experiment and analysis section gives the experimental result of proposed scheme. The security and privacy of proposed scheme are discussed in Discussion section, and finally, the paper is concluded in Conclusion and future work section.

\section{Related works}

Among the techniques are used to protect digital intellectual property, digital watermarking of information hiding technology is the most commonly used. The creation of digital watermarking dated back to 1954, when Emil Hembrooke of Muzak applied for a patent for a watermarking method on musical composition by intermittently applying a narrowband trap filter with a central frequency of $1 \mathrm{kHz}$ and inserting a verification code. This system was used by Muzak until 1984. Research on digital watermarking began in the early 1990s. The concept of digital watermarking was first introduced at the International Conference on Image Processing in 1994 [2], and then the First International Symposium on Information Hiding was held in the UK in 1996 [3]. At the Third International Symposium on Information Hiding in 1999, digital watermarking dominated the conference, with 18 of the 33 papers were devoted to the study of digital watermarking. Digital watermarking has evolved towards copyright protection for any types of digital files. For example, IBM is particularly active in digital watermarking. It provides digital watermarking in its "Digital Library" software. The IBM's latest research currently developed a watermarking model for deep neural network (DNN) models presented in 2018, which contained three watermarking algorithms for copyright authentication of DNN models. Except using digital watermarking for protection, PetaPixel develops Blockkai which uses the Bitcoin blockchain to protect copyright, and Baidu's "Totem" project uses blockchain technology to solve the pain point of image copyright [4]. Tencent Cloud uses blockchain technology to launch the "Zhixin Chain" to solve the problem of original content protection. Byte Jump's Tuchong enters into a deep partnership with Ant Blockchain of Ant Gold. Tuchong become the leading blockchain platform for the identification and ownership of photographers and the original works on Tuchong platform can be checked on the chain [5]. 
In recent years, scholars from all over the world have made great progress in the study of digital watermarking on images. KOBAYASHI Atsushi, HASEGAWA Akria and et al. propose that digital watermarking algorithms can be embedded in edge devices as a complement to other permission management and they investigate the application of digital watermarking to both still images and moving images [6]. He Fang studies on waveletbased high-frequency and low-frequency methods for inserting watermark and tests their anti-compressibility for watermark extraction. His experiments show that the insertion of watermark in the high frequency domain maintains the original quality better, and the insertion of watermark domain makes the robustness better in the low-frequency [7]. Zairong WANG, Babak SHIRI's innovatively use fractional logistic mapping for generating chaotic sequences, then encrypt and insert the watermark into the original image. Because the fractional order of the encrypted image is within $(0,1]$, the key space is increased [8]. Their research provides a robust and safe watermarking method for protecting the copyright of hardware, image and other electronic documents [8]. Yuan-Min Li and Deyun Wei propose a watermarking algorithm with double encryption based on cosine transform and fractional fourier transform in the invariant wavelet domain. Their work innovatively utilises image magnification technique to pre-process the host image to enhance embedding capacity of the watermarking algorithm. They use the redistribution-invariant wavelet transform and the discrete cosine transform to obtain the hybrid domain, and they also propose a multi-parameter particle swarm optimisation algorithm, which is used to obtain the optimal embedding factor matrix [9].

Zero-watermarking [10] has become a focus of research in recent years since it was proposed by WenQuan in 2003, as it did not make any modification to the original digital file. Zhiqiu Xia and Xingyuan Wang et al. propose multiple zero-watermarking scheme based on local quaternion polar harmonic Fourier moments (QPHFMs) for colour medical images, and the construction of multiple zero-watermarking effectively improves reliability and resists complex out-of-step attacks, such as clipping [11]. Hongfei Xue and Wanda Chi propose a more robust zero-watermarking algorithm based on NSST, DCT and Hessenberg decomposition. They innovatively generate a zero-watermark by performing XOR between the QR code and the characteristic matrix of a colour image [12]. In recent years, the combination of deep learning and watermarking algorithm has been also a key research area. Ali Amiri and Satter Mirzakuchaki propose an intelligent watermarking model based on NSCT-SVD. They innovatively use PSO-GAAI algorithm, and the obtained PSO and PSO-GA algorithms with larger SF have higher stability, then low frequency coefficient is fed back to the SVD through NSCT and SWT so that the values of model visual transparency and PSNR will increase [13].

Video is a very important part of the digital media. Currently, there are three types of schemes about video watermarking. The first way is the watermarking scheme based on original video, which embeds the information of watermark into the original video carrier. The second way is based on the video encoding process. The compression standards of mainstream video include the MPEG series of ISO/IEC and the H.264 series of ITU-T [14,15], and the key technology for these compression standards to achieve a high compression ratio is predictive coding and transform coding. The disadvantage of the second way is the presence of cumulative errors which can have an adverse effect on video quality [16]. The third way is based on the compression domain. Because the video is currently transmitted and stored in a compressed form. The advantage of this way is that the embedded watermarking doesn't need to be reencoded and decoded, so it can reduce computational complexity through this way. The disadvantage is that the low redundancy of the compressed data limits the amount of data that can be watermarked, and the embedding strength is limited by the encoding of the video data. Y Sun and J Wang remedy the shortcomings of the existing watermarking algorithms on H.264/SVC framework. Their research uses DCT (the discrete cosine transforms) and an invisible watermarking framework with scalable watermarking algorithms to accommodate the multi-resolution of H.264 / SVC, and they design control mechanisms of key to select sub-blocks adaptively from macroblocks [17]. Their proposed method achieves no less than $41 \mathrm{~dB}$ peak signal-to-noise ratio (PSNR) and a normalised correlation (NC) of >0.85 [17]. Yicheng Qiu and Feng Xue et al. propose a novel method of video watermarking for HEVC (High Efficiency Video Coding) that the blocks for watermarking embedding are selected based on the temporal and spatial characteristics of the compressed video, which minimizes the synchronization error and can well prevent collusion attacks [18]. Besides, the public key is extracted from features of compressed domain to select the blocks for embedding, and the watermark is embedded on the blocks which reduces the computational overhead [18]. 


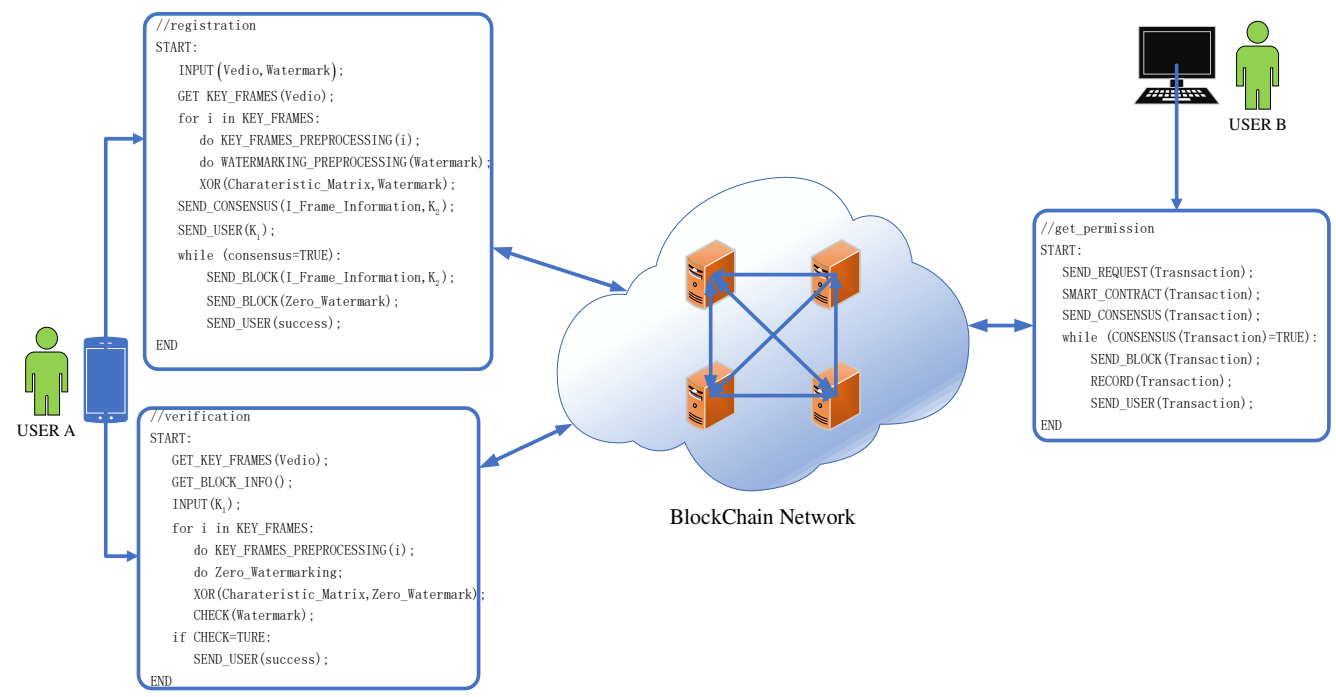

Fig. 1 Framework of the protection system

\section{Scheme}

\section{Structure of the protection system for video} copyright

Firstly, the main processes of registration and permission transactions about video copyright are as follows:

Step 1. User A uploads a video and a colour image which authenticates identity through the terminal. Step 2. Pre-process the video data and extract the key-frames by distance threshold clustering.

Step 3. Construct zero-watermarks on extracted key-frames.

Step 4. Collect zero-watermarks, infor-mation of key-frames and Key $K_{2}$, then package these data.

Step 5. Upload the packaged data to the blockchain network and send it to the consensus engine.

Step 6. The consensus engine sends the block to the blockchain network when the block's verification is correct and the nodes reach a consensus in the blockchain network, otherwise, the system will delete the block.

Step 7. Blockchain network check information of block (such as block height) and store block and table data.

Step 8. Return the Key $K_{1}$ to User A.

Step 9. User B initiates a transcation request for the permission to use or own the video.

Step 10. Blockchain network receives the transaction request and automatically executes the transaction through smart contract, then packages the result of the transaction to the consensus engine.

Step 11. Once each user reached consensus on a transaction, the consensus engine will send the transaction to the blockchain network, which stores the error-free transaction.

Step 12. Return a message about successful transaction to User B.

Secondly, the main processes for certification about video copyright are as follows:

Step 1. In order to authenticate the video, User A need to provide Key $K_{1}$ and the block hash to match other information on the blockchain to get the information of key-frames, zero-watermarks and Key $K_{2}$ on the chain.

Step 2. Use the watermark extraction algorithm to return a colour watermark which can authenticate the identity and complete the authentication.

Step 3. User B can simply check ledger on the blockchain network when he completes the transaction to prove his copyright of the video. The above processes are described in Fig. 1.

\section{Key-frames extraction method based on distance threshold clustering}

Step 1. Input video samples and process the video library.

Step 2. Convert unstructured dynamic video into a static sequence of video frames.

Step 3. Process the samples initially and extract the feature vectors.

Step 4. Calculate the inter-frame similarity and distance threshold according to the extracted feature vectors to complete the clustering.

Step 5. Judge key-frames based on the clustering results

Step 6. Output the extracted key-frames.

Steps are shown in Fig. 2. 


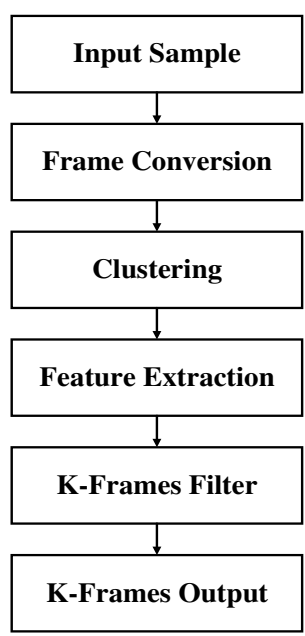

Fig. 2 Process of key-frames extraction

\section{Feature extraction}

The selection of feature is very important to the expression of key-frame and the completion of effective clustering. Colour and texture features are common image features. At present, most of the key-frame extraction methods are based on clustering use colour histogram, but the feature of colour histogram cannot describe colour space distribution between adjacent pixels. As a local feature of an image, texture feature does not depend on colour and brightness. It not only reflects the grey level statistics of the image, but also reflects the spatial distribution information and structure information of the image. Its grey level invariability and rotation invariant can cleverly avoid experimental errors caused by significant changes in light. Therefore, in this paper, the completed LBP (CLBP) [19] descriptor of the improved local binary patterns (LBP) [19] is chosen to handle the texture features. CLBP texture descriptor [20] is an extended texture descriptor based on LBP descriptor [21], which effectively describes the missing information of LBP type in order to obtain better performance of texture classification.

Usually, given a central pixel and other $\mathrm{P}$ neighbourhood values, the local difference vector is expressed as $\left[d_{0} \ldots \ldots . d_{p-1}\right], d_{p}=g_{p}-g_{c} \cdot s_{p}$ and $m_{p}$ are $d_{p}$ using local difference symbols, that is, symbol and amplitude value of local difference sign-separation-magnitude transform (LDSMT). The above content is shown as follows:

$$
\begin{gathered}
\mathrm{d}_{p}=s_{p} \cdot m_{p} \text { and }\left\{\begin{array}{c}
s_{p}=\operatorname{sign}\left(\mathrm{d}_{p}\right) \\
m_{p}=\left|\mathrm{d}_{p}\right|
\end{array}\right. \\
s_{p}=\left\{\begin{array}{c}
1, d_{p} \geq 0 \\
-1, d_{p}<0
\end{array}\right.
\end{gathered}
$$

The above formulas cannot be used directly as

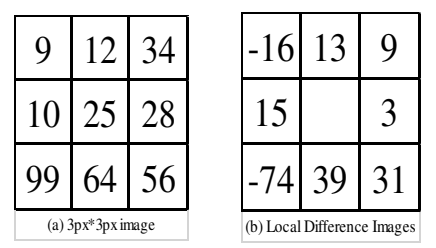

Fig. 3 Description of CLBP algorithm I
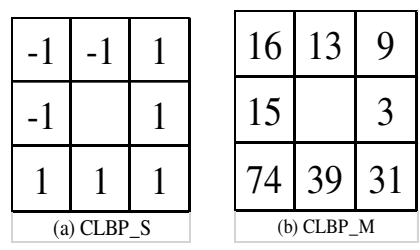

Fig. 4 Description of CLBP algorithm II

a descriptor because it is sensitive to light, rotation, noise, etc. The difference between the neighbouring pixels and the central pixel is divided into a sign component and a gradient component and is obtained by multiplying the two together, denoted as CLBP_S and CLBP_M. For example, Fig. 3a is $3 \times 3$ sampling block, which can be divided into Fig. $4 \mathrm{C} \times \mathrm{Fig} .4 \mathrm{~d}$, and its difference can be represented by Fig. 3b. Fig. 4a is sign components, and Fig. 4b is gradient components. Actually, CLBP_S is the traditional LBP, and the two coding methods are the same. 1 in Fig. 4a is equal to 0 in the traditional LBP. What's more, the CLBP_S is more capable of describing texture than the CLBP_M. Formula of CLBP_S is shown as follows:

$$
a B P_{-} s_{P, R}=\sum_{P=0}^{P-1} s\left(s_{p}\right) 2^{p}
$$

and

$$
s(x)=\left\{\begin{array}{l}
1, x \geq 0 \\
0, x<0
\end{array}\right.
$$

As can be seen from Fig. 4b, CLBP_M is a continuous value. In order to harmonise the encoding with CLBP_S, it needs to be converted to binary encoding as well. Inspired by CLBP_S, this paper follows the following encoding for them, $\mathrm{c}$ is an adaptive threshold, and the value of $c$ is taken as the mean value of $m_{p}$ in the whole image. $m_{p}$ is the absolute value accumulation of gradient differences between neighbourhood pixels and centre pixels (or the average of them, the final results of comparison are equal). Formula of CLBP_M is shown as follows:

$$
a B P_{-} M_{P, R}=\sum_{p=0}^{p-1}\left(m_{p}, c\right) 2^{p}
$$

and

$$
t(x, c)=\left\{\begin{array}{l}
1, x \geq c \\
0, x<c
\end{array}\right.
$$




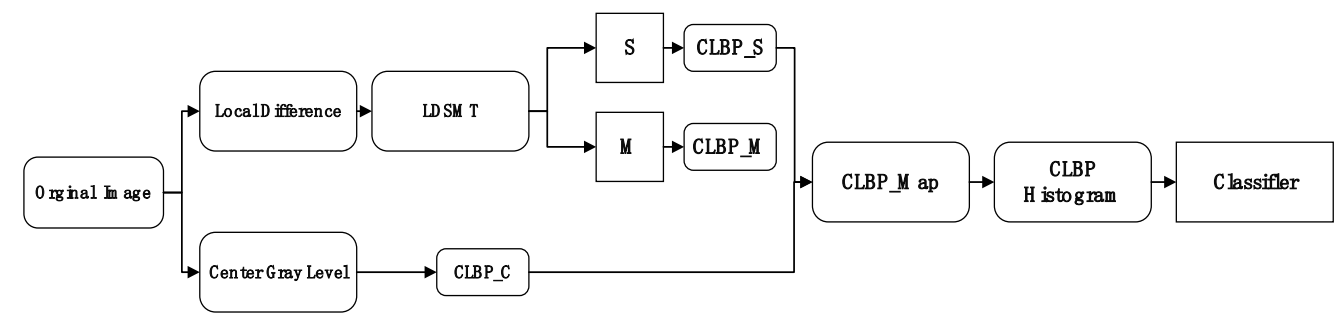

Fig. 5 CLBP algorithm

The central pixel represents the local grey level and also contains the local grey level discriminant information. In order to effectively combine it with CLBP _ M and CLBP _ S, we carry out the following encoding:

$$
a B P_{-} C_{P, R}=t\left(g_{c}, c_{l}\right)
$$

and

$$
t(x, c)=\left\{\begin{array}{l}
1, x \geq c \\
0, x<c
\end{array}\right.
$$

In Eq.7, $g_{c}$ represents the centre pixel grey value, $c_{\text {l }}$ represents the average grey value of the whole image. By comparing the centre pixel and the average pixel value of the whole image to binary encoding.

The process of CLBP algorithm is shown in Fig. 5. After the above calculation, the three descriptors CLBP _ S, CLBP_ M, CLBP _ C are all generated, which can be fused in the form of series, parallel or series-parallel histogram, and can achieve more effective rotation invariant classification ability than the traditional LBP.

\section{Inter-frame similarity calculation}

Distance measurement is used to measure the distance between individuals in space. The farther the distance is, the greater the difference between individuals is, and the lower the similarity is. Chisquare distance is the most common measure to measure the difference between two individuals, and its calculation is simple. Therefore, in this paper we use chi-square distance to represent the similarity between frames. The smaller the chisquare distance is, the higher the similarity between frames is. The chi-square distance between any two frames is defined as follows:

$$
\operatorname{dist}\left(F_{a}, F_{b}\right)=\sum_{i=1}^{n} \frac{\left(F_{a}[i]-F_{b}[i]\right)^{2}}{F_{a}[i]+F_{b}[i]}
$$

In Eq. 9, $F_{a}[i]$ and $F_{b}[i]$ represent the values of image a and image $b$ on the i-th bin. Respectively, $n$ is the total number of video frame histogram bins, and dist $\left(F_{a}, F_{b}\right)$ represents the inter-frame similarity.

\section{Distance threshold calculation}

The distance threshold directly affects the number of clusters, thereby affecting the extraction effect of key-frames. If the threshold is too small, it is easy to extract too many key-frames, resulting in information redundancy. If the threshold is too large, the extracted key-frames cannot represent the main content of the lens. According to the idea of data density sampling [23], the distance threshold is defined as follows:

$$
d=\frac{2 c}{N(N-1)} \sum_{i=1}^{N-1} \sum_{j=i+1}^{N} d i s t\left(F_{i}, F_{j}\right)
$$

In Eq. 10, $N$ is the total number of video frames, and $N(N-1) / 2$ is the number of chi-square distances between two $\mathrm{N}$ frames. $\mathrm{c}$ is a constant. A large number of experiments show that the value of 0.25 in this algorithm is better. $d$ is the distance threshold of clustering algorithm obtained by density sampling. By this method, different thresholds can be selected for different videos, which reflects the adaptability of the threshold. The threshold selected by this method will be small but not too small, and appropriate small threshold can obtain more initial clustering, which is conducive to cluster centre merging and secondary clustering.

\section{Algorithm description}

All the steps of algorithm description are shown as follows:

Step 1. Sample the data set to determine the distance threshold d.

Step 2. Initial clustering of data sets based on threshold and similarity, and then determine the initial number of clusters $\mathrm{k}$ and cluster centre set C.

Step 3. Use K-means algorithm to optimize the initial clustering centre, then get new sets of clustering centre $\mathrm{G}$.

Step 4. Use the sequential clustering idea [24] to merge the closer classes in $\mathrm{G}$, then determine the final $\mathrm{k}$ and cluster centre set and cluster, and then complete the clustering centre self-determination.

Process of clustering algorithm is shown in Fig. 6. After clustering, select all the nearest frames from the clustering centre to be the key-frames of the video. 


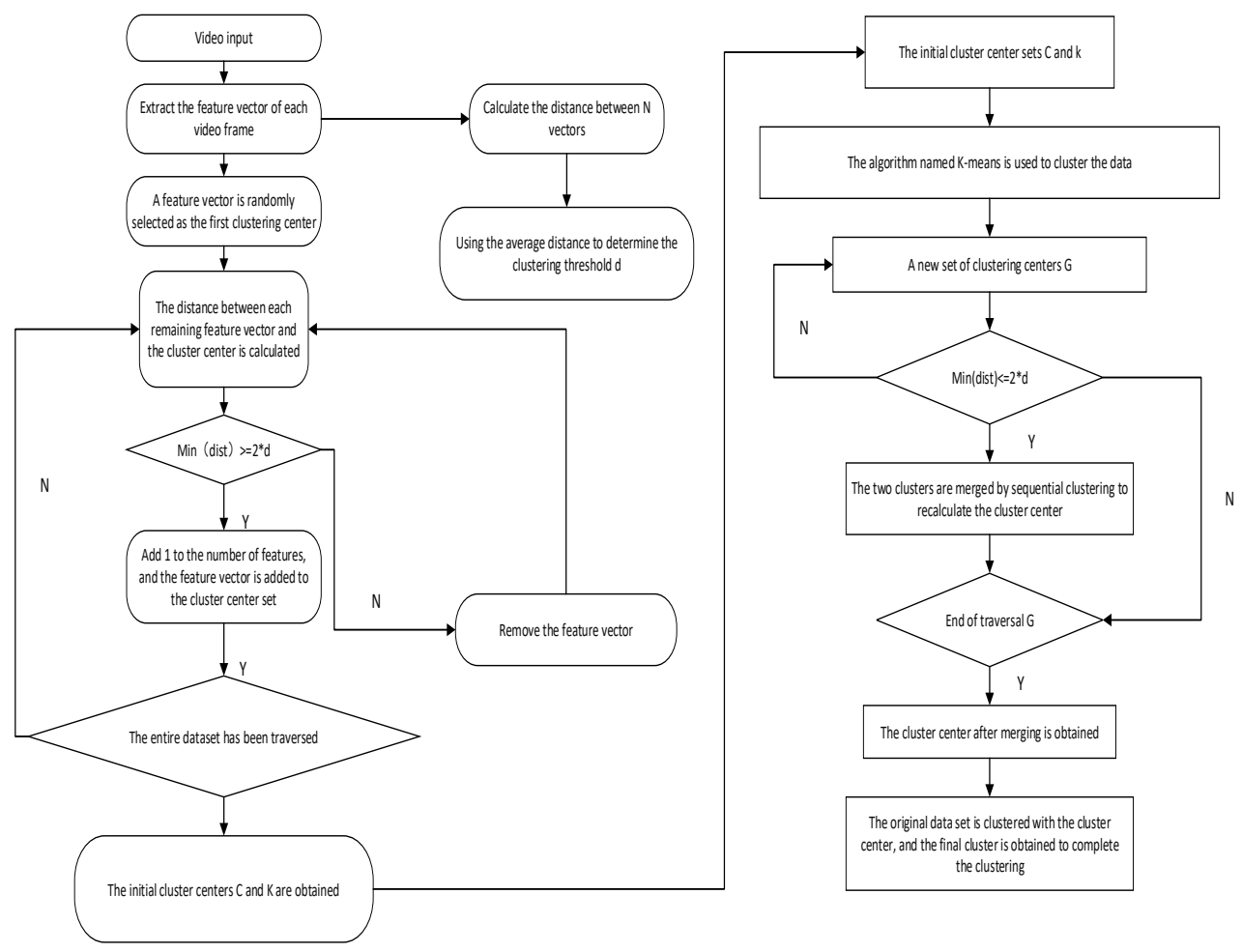

Fig. 6 Process of clustering algorithm

\section{Zero-watermarking algorithm for key-frames based on NSCT and SVD Preliminary knowledge}

After the digital image has been SVD [25-27], the corresponding orthogonal matrix represents the geometric structure of the image while the corresponding singular matrix represents the image luminance information. Define a field of real numbers is $\mathrm{R}$. Assume the digital image matrix is $\mathrm{A}$, and $\mathrm{A} \in R_{N \times N}$, Orthogonal matrix $\mathrm{U}$ $\in R_{N \times N}$, orthogonal matrix $\mathrm{V} \in R_{N \times N}$, diagonal matrix $S \in R_{N \times N}$, so A can be expressed as:

$$
A=S \times U \times V^{T}
$$

$\mathrm{U}$ is the left SVD orthogonal matrix and V is the right SVD orthogonal matrix. Satisfy the conditions respectively $U \times U^{T}=1, V=V^{-1}$. In that $S$ :

$$
S=\left(\begin{array}{ccc}
\lambda_{1} & 0 & 0 \\
0 & \ddots & 0 \\
0 & 0 & \lambda_{r}
\end{array}\right)
$$

A special SVD's value in the $S$ matrix is usually much larger than other SVD, and SVD usually represent the magnitude of the overall energy of A [28].

The Arnold Transform [29-31] can change the position of pixels and eliminate correlations between pixels or content in a carrier image, thus improving image security. The special Arnold Transform is the Simplest kind of Arnold Transform. The formula for operation of matrix is:

$$
\left(\begin{array}{l}
x^{\prime} \\
y^{\prime}
\end{array}\right)=\left[\begin{array}{ll}
1 & 1 \\
1 & 2
\end{array}\right]\left(\begin{array}{l}
x \\
y
\end{array}\right) \operatorname{nod}(N)
$$

The special Arnold Inverse Transform makes it easy to recover the transformed image. The formula for operation of matrix is:

$$
\left(\begin{array}{l}
x \\
y
\end{array}\right)=\left[\begin{array}{cc}
2 & -1 \\
-1 & 1
\end{array}\right]\left(\begin{array}{l}
x^{\prime} \\
y^{\prime}
\end{array}\right) \bmod (N)
$$

$\left(x^{\prime}, y^{\prime}\right)$ denotes the position of $(x, y)$ at the pixel point after the transformation and $\mathrm{N}$ denotes the size of the image involved in the transformation.

\section{Watermark generation}

After using the key-frames extraction method based on distance threshold clustering, a series of key-frames are selected from the video which represent the feature information of the whole video. The zero-watermarking algorithm is that extracting the feature information of these keyframes and combining with the watermark later. The detailed steps are as follows:

Step 1. Output the information of Frame I after selecting the key-frames in the video frame sequence, then get key-frames shapes of $M \times N \times 3$.

Step 2. Perform RGB separation of the key- 


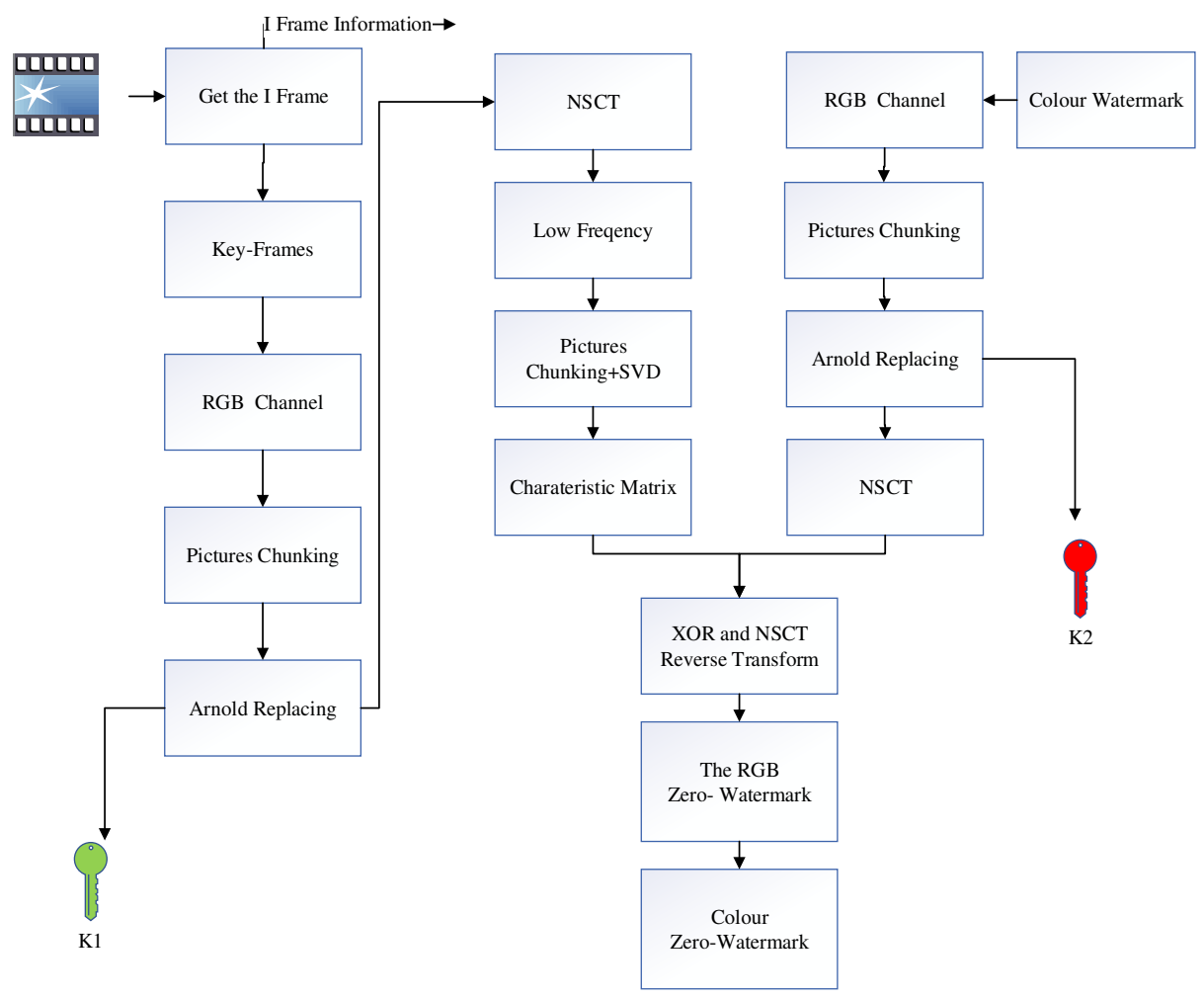

Fig. 7 Process of watermark generation

frames and get $I_{B}, I_{G}, I_{B}$ after separating. Then make $I_{B}, I_{G}, I_{B}$, the three Frame I into $A \times B$ non-overlapping chunks (Operations of the separated three Frames I are the same and we will only introduce one in the following). Suppose the values of $\mathrm{M}$ and $\mathrm{N}$ are both 512, and the values of $\mathrm{A}$ and $\mathrm{B}$ are both 128 . The chunking method is as follows:

$$
B_{i, j}=\frac{I_{k}^{M \times N}}{A \times B}, k \in\{R, G, B\}, i, j \in\{1,2,3,4\}
$$

Step 3. Do Arnold Transform on each sub-block, and the disordering key is Key $K_{1}$. Assume that the chunked sub-blocks are transformed according to Eq. 13. The Arnold Transform will break up the pixel points of the image in the RGB channels.

Step 4. Do NSCT for each sub-block $B_{i, j}$ after scrambling. The NSCT yields low-frequency information $c A_{, j}$ for each sub-block containing point singularities and curve singularities. Following the assumptions in Step 2, The size of $c A_{j}$ is $32 \times 32$.

Step 5. Subdivide the low frequency component $c A_{j, j}$ again and subdivide $c A_{, j}$ into sub-blocks of $4 \times 4$ size. The chunking method is as follows:

$c B_{m n}=\frac{c A_{j}}{8 \times 8}, m n \in\{1, \ldots, 8\}, i, j \in\{1, \ldots, 4\}$

Step 6. Do SVD [32] on each sub-block according to Eqs. 8 and 9. The method is as follows:

$$
\left.\left[U_{i, j}, S_{i, j}, V_{i, j}\right]=S \operatorname{SQ} c A_{, j}\right)
$$

Owing to the highest bit of each singular value of each sub-block does not change under a certain range of attacks, the maximum singular value of each singular value matrix is chosen to construct the characteristic matrix $X$. After SVD, characteristic matrixes $\left\{X_{R}, X_{G}, X_{B}\right\}$ can be get. Characteristic martix $\mathrm{X}$ is as follow:

$$
x=\left[\begin{array}{ccc}
f_{1,1} & \cdots & f_{1,32} \\
\vdots & \ddots & \vdots \\
f_{32,1} & \cdots & f_{32,32}
\end{array}\right]
$$

Step 7. Process meaningful watermark image shapes of $m \times n \times 3$. After the RGB colour separation, get the $I_{R}^{\prime}, I_{G}^{\prime}, I_{B}^{\prime}$, then do Arnold Transform on $I_{B^{\prime}}, I_{G}^{\prime}, I_{B}^{\prime}$ and its the distorting key is Key $K_{2}$. Assume the disordered frames are $W_{a}^{\prime}, w_{c}^{\prime}, W^{\prime}$.

Step 8. Do NSCT on $W_{a}^{\prime}, w_{a}^{\prime}, W_{3}^{\prime}$, then get both high frequency part $H_{R}{ }^{\prime}, H_{G}{ }^{\prime}, H_{B}{ }^{\prime}$ and the low frequency part $L_{R}{ }^{\prime}, L_{R}{ }^{\prime}, L_{R}{ }^{\prime}$. Run XOR $\left\{H_{R}{ }^{\prime}\right.$, $\left.H_{G}{ }^{\prime}, H_{B}{ }^{\prime}\right\}$ and $\left\{L_{R}{ }^{\prime}, L_{R}{ }^{\prime}, L_{R}{ }^{\prime}\right\}$ with each of the three channels of the characteristic matrixes $\left\{X_{R}\right.$, $\left.X_{G}, X_{B}\right\}$ obtained in Step 6. The method is as follows:

$$
\begin{array}{r}
\left.Z_{k+1}=X O R X_{k}, H_{k}{ }^{\prime}\right), K \in\{R, G, B\} \\
\left.Z_{k}=X O R X_{k}, L_{k}{ }^{\prime}\right), K \in\{R, G B\}
\end{array}
$$

Step 9. Do the inverse transform of NSCT on $Z_{k+1}$ 


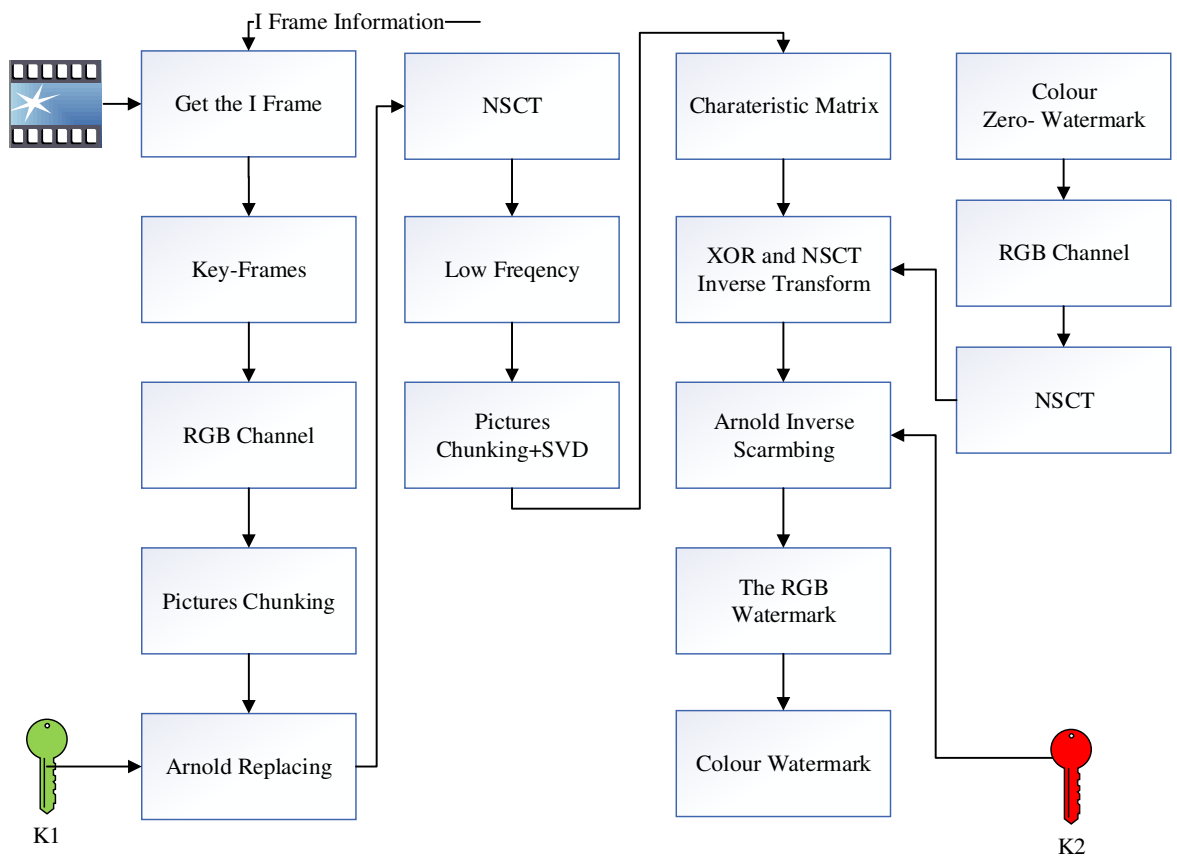

Fig. 8 Process of watermark extraction

and $Z_{k}$, then composite RGB to produce a colour zero-watermark. The method is as follows:

$$
\begin{gathered}
Z_{K H}+Z_{K}=Z_{K}, K \in\{R, G, B\} \\
Z_{R, G B}=Z_{R}+Z_{G}+Z_{B}
\end{gathered}
$$

All the above Steps are shown in Fig. 7.

\section{Watermark extraction}

Step 1. Based on the information of the Frame I, obtain key-frames.

Step 2. Follow Step 2 of watermark generation for RGB separation and chunking.

Step 3. Use $K_{1}$ to do Arnold Transform like Step 3 of watermark generation for each of the chunked sub-blocks.

Step 4. Follow Step 4, Step 5 and Step 6 of watermark generation. After those Steps, characteristic matrixes $X_{R}{ }^{\prime}, X_{G}{ }^{\prime}, X_{B}{ }^{\prime}$ are generated.

Step 5. Process colour zero-watermark shapes of $m \times n \times 3$. After the RGB separation, the separated frames are $I_{R} ", I_{G} ", I_{B} "$. Do NSCT on $I_{R} ", I_{G} ", I_{B} "$, then get both high frequency part $H_{R} ", H_{G} ", H_{B}$ " and the low frequency part $L_{R} ", L_{R} ", L_{R} "$. Run XOR $\left\{H_{R} ", H_{G} ", H_{B} "\right\}$ and $\left\{L_{R} ", L_{R} ", L_{R} "\right\}$ with each of the three channels of the characteristic matrixes $\left\{I_{R}{ }^{\prime \prime}, I_{G}{ }^{\prime}\right.$, $\left.I_{B} "\right\}$. The method is as follows:

$$
\begin{gathered}
\left.V_{k-1}{ }^{\prime}=X O R X_{k}{ }^{\prime}, H_{K}{ }^{\prime \prime}\right), K \in\{R, G, B\} \\
\left.V_{k}{ }^{\prime}=X O R X_{k}{ }^{\prime}, L_{K}{ }^{\prime \prime}\right), K \in\{R, G, B\}
\end{gathered}
$$

Step 6. Do the inverse transform of NSCT on $Z_{K+}{ }^{\prime}$ and $Z_{K}{ }^{\prime}$.

$$
V_{K-1}{ }^{\prime}+V_{K}{ }^{\prime}=V_{K}{ }^{\prime}, K \in\{R, G, B\}
$$

Step 7. Use the Key $K_{2}$ which is kept by the owner of video copyright to do the Arnold Inverse Transform, then we get $Z_{R, G B}$ ' from $V_{R, G B}$ ' by Arnold Inverse Transform. The method similar to Eq. 14. Finally, composite RGB to produce a colour watermark. The method is as follows:

$$
Z_{K}{ }^{\prime}=Z_{R}{ }^{\prime}+Z_{G}{ }^{\prime}+Z_{B}{ }^{\prime}
$$

All the above Steps are shown in Fig. 8.

\section{Experiment and analysis}

In order to verify the reliability of the proposed scheme, two parts are tested in this part. One is the reliability of zero-watermarking algorithm, the other is the efficiency of blockchain system.

\section{Experimental environment}

All parameters of experimental environment are shown in Table 1. 
Table 1 Experimental environment parameters

\begin{tabular}{ll}
\hline $\begin{array}{l}\text { Environmental } \\
\text { parameters }\end{array}$ & Config \\
\hline $\begin{array}{l}\text { Operating } \\
\text { environment } \\
\text { Host Environment }\end{array}$ & Windows 10 \\
Database & MySQL 8.0.18 \\
Blockchain platforms & Fisco Bcos \\
RAM & $8 \mathrm{~GB}$ \\
Processor information & Intel i5,2.4 GHz CPU \\
\hline
\end{tabular}

\section{Analysis on blockchain system}

This part tests response time of blockchain system, so as to illustrate the feasibility and reasonableness of this system which combines with blockchain.

Besponse time of blockchain system is one of the important indicators of system performance. The main functions of the system in proposed scheme are divided into on-chain data entry and off-chain data management. The off-chain data is processed by each role subject in a distributed and autonomous manner, which does not have an impact on the response time of the traceability system, and the speed of traceability information on the chain is the key to influence the response time of the system.

Fig. 9 shows the relationship between the amount of data on the chain and the system response time. When the data on the chain increases from $1 \mathrm{G}$ to $8 \mathrm{G}$, the system response time increases from $231 \mathrm{~ms}$ to $511 \mathrm{~ms}$. The results show that when the amount of data increases, the response time of the system increases with a linear trend. The delay of the proposed blockchain system is acceptable within the normal range in the actual protection system of video copyright.

\section{Analysis on key-frames extraction}

This part tests the effectiveness of video keyframes extraction algorithm by K-means clustering algorithm. The video testing set is comprehensive, including landscapes, advertisement, animation clips and news. This experiment has the following definitions:

Recall ratio is $R$ and Precision ratio is $P$. Correctly detected key-frame is CF. Missed detected key-frame is MF. False detected keyframe is DF. R and P are calculated as follows:

$$
\begin{aligned}
& R=\frac{C F}{O F+M F} \\
& P=\frac{C F}{O F+D F}
\end{aligned}
$$

From the experimental data in Table 2, it can be seen that key-frames extraction algorithm works well for various videos. Although a small number of missed detected key-frames and false detected key-frames exist, the video key-frames extraction algorithm can reduce the rate of missed detections and false detections.

Table 2 Video key-frames extraction experimental results

\begin{tabular}{ccccc}
\hline & Landscapes & Advertisement & $\begin{array}{l}\text { Animation } \\
\text { clips }\end{array}$ & News \\
\hline $\begin{array}{c}\text { Total } \\
\text { frames }\end{array}$ & 115 & 1254 & 15324 & 26987 \\
CF & 14 & 135 & 735 & 1452 \\
Df & 1 & 7 & 52 & 182 \\
MF & 0 & 2 & 23 & 85 \\
R /\% & 100 & 98.54 & 96.97 & 94.47 \\
P /\% & 93.33 & 95.07 & 93.39 & 88.86 \\
\hline
\end{tabular}

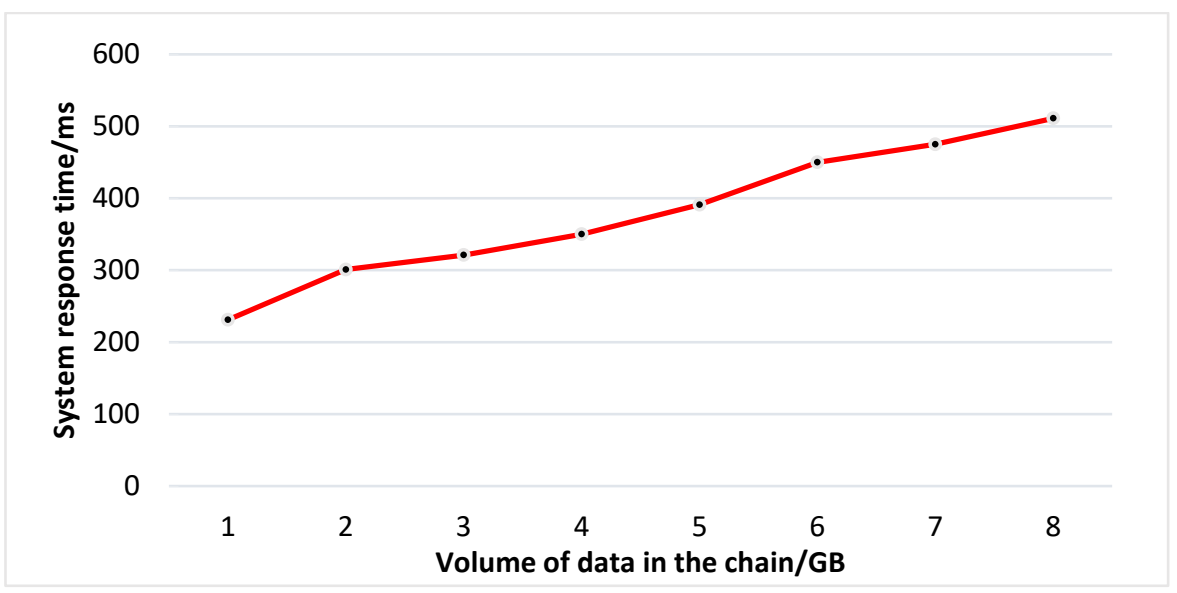

Fig.9 System response time and on-chain data volume testing 


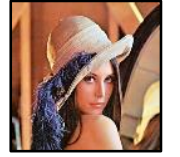

(a)len

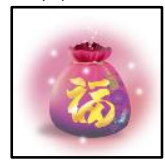

(d)bag

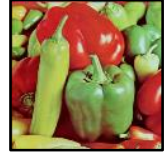

(b)vegetable

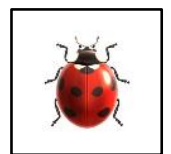

(e)ant

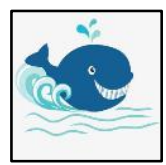

(c)animal

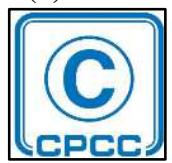

(f)CPCC

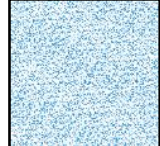

(g)zero-watermark

Fig. 10 Images, copyright watermarking and zero-watermark

Table 3 NC of zero-watermark between different images

\begin{tabular}{llclll}
\hline $\begin{array}{l}\text { Host } \\
\text { Image }\end{array}$ & Lena & Vegetable & Bag & Ant & Animal \\
\hline Lena & 1.0000 & 0.5769 & 0.5125 & 0.4643 & 0.5571 \\
Vegeta & 0.5769 & 1.0000 & 0.4517 & 0.4943 & 0.3753 \\
ble & & 0.4517 & 1.0000 & 0.5360 & 0.4777 \\
Bag & 0.5125 & 0.4943 & 0.5360 & 1.0000 & 0.5570 \\
Ant & 0.4643 & 0.457 \\
Animal & 0.5571 & 0.3753 & 0.4777 & 0.5570 & 1.0000 \\
\hline
\end{tabular}

Analysis on video zero-watermarking algorithm Five images are selected to instead key-frames in experiments, which are Fig. 10a-10e. Fig. 10f is defined as copyright watermark in experiment. Fig. $10 \mathrm{~g}$ is a generated example of zerowatermark. All the figures are shown in Fig. 10.

\section{false alarm}

In this part, we discuss false alarm on the proposed video zero-watermarking algorithm in this paper.

Most of traditional zero-watermarking algorithms have the problem of false alarm, because the base space of SVD decomposition is related to the content of the image, and there is no one-to-one correspondence between the singular value vector and the image.

In this experiment, we use the five images which are Fig. 10a-10e to generate the corresponding zero-watermark with the copyright watermark, and then calculate normalized correlation (NC) between the five zerowatermarks. $\mathrm{NC}$ value reflects false alarm rate. The experimental results are shown in Table 3.

According to Table 3, the proposed zerowatermaking algorithm can effectively avoid false alarm in this paper.
Table 4 Comparation between DWT-SVD and NSCT-SVD

\begin{tabular}{lll}
\hline \multicolumn{1}{c}{ Attack Types } & DWT-SVD & NSCT-SV \\
\hline $\begin{array}{l}\text { Sault and pepper } \\
\text { noise (0.03) }\end{array}$ & 0.9669 & 0.9870 \\
$\begin{array}{l}\text { Gaussian noise (0.03) } \\
\begin{array}{l}\text { Multiplicative noise } \\
\text { (0.03) }\end{array}\end{array}$ 0.9643 & 0.9785 \\
$\begin{array}{l}\text { Median filtering (5*5) } \\
\text { Mean filtering }(5 * 5)\end{array}$ & 0.9709 & 0.9915 \\
& 0.9752 & 0.9966 \\
Wiener filtering $(5 * 5)$ & 0.9781 & 0.9944 \\
\hline
\end{tabular}

\section{Robustness}

In this part, we discuss robustness on the proposed zero-watermarking algorithm in this paper from three parts which are noise attack, filtering attack, geometric attack.

Before completely attacking the proposed zerowatermarking, we select Fig. 10a to compare traditional DWT-SVD and NSCT-SVD in resisting conventional attacks, and the similarity of copyright watermark extracted after attacks is calculated. According to Table 4, NSCT-SVD has greater superiorities under noise attacks, filtering attacks than DWT-SVD.

(1) Noise attack

As seen from Figs. 11, 12 and 13, the watermarks maintian good visual stability after extraction under noise attacks on Fig. 10e.
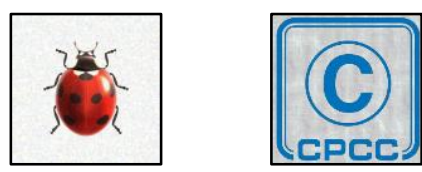

Fig. 11 Attack of salt and pepper noise (0.03) and the extracted watermark
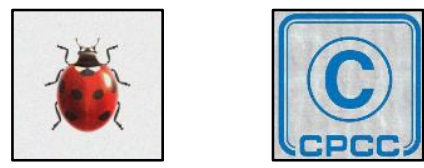

Fig. 12 Attack of gaussian noise (0.03) and the extracted watermark

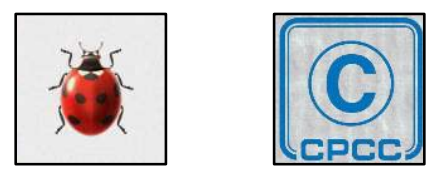

Fig. 13 Attack of multiplicative noise (0.03) and the extracted watermark 
Table 5 NC values obtained from watermark of the Fig. 9a-e under noise attacks

\begin{tabular}{ccccccc}
\hline Attack Types & Parameters & Lena & Vegetable & Animal & Bag & Ant \\
& & & & & & \\
\hline \multirow{2}{*}{ Sault and pepper noise } & 0.01 & 0.9958 & 0.9957 & 0.9946 & 0.9942 & 0.9963 \\
& 0.03 & 0.9870 & 0.9868 & 0.9864 & 0.9875 & 0.9885 \\
Gaussian noise & 0.01 & 0.9801 & 0.9811 & 0.9807 & 0.9809 & 0.9789 \\
& 0.03 & 0.9785 & 0.9767 & 0.9777 & 0.9789 & 0.9765 \\
Multiplicative noise & 0.05 & 0.9667 & 0.9639 & 0.9656 & 0.9645 & 0.9658 \\
& 0.01 & 0.9981 & 0.9973 & 0.9978 & 0.9975 & 0.9979 \\
& 0.03 & 0.9915 & 0.9910 & 0.9946 & 0.9934 & 0.9961 \\
& 0.05 & 0.9856 & 0.9838 & 0.9919 & 0.9894 & 0.9876 \\
\hline
\end{tabular}

As shown in Table 5, the NC values obtained from watermark under noise attacks are all very high. The highest NC value is 0.9979 and the lowest is 0.9645 , with the average value is
0.9812. Due to the combination of NSCT and SVD, the change in the low frequency coefficients of each sub-block is small under noise attacks. The results show the algorithm is

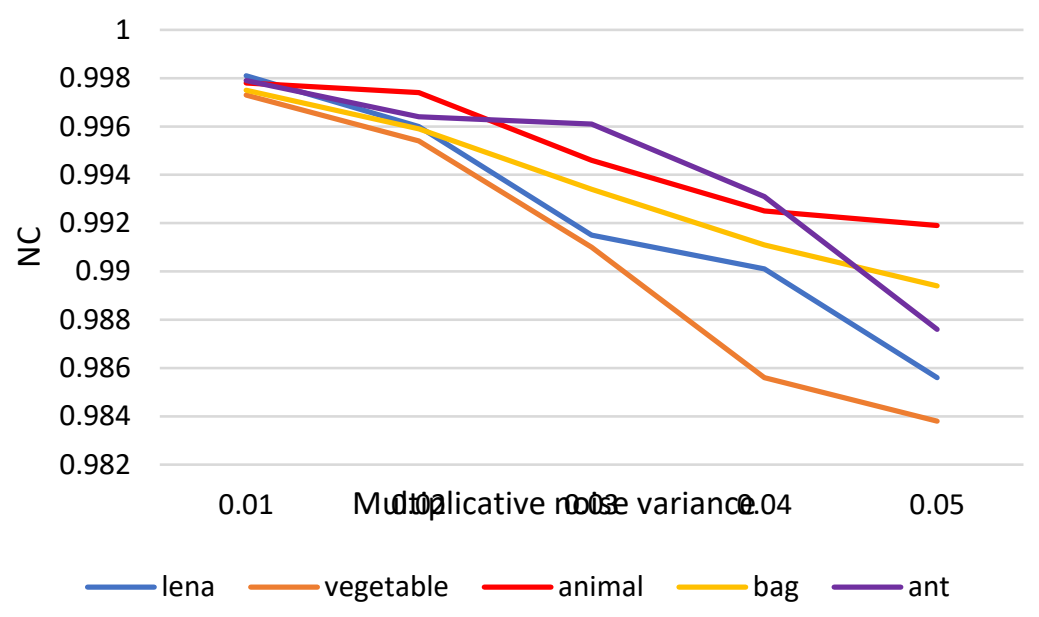

Fig. 14 Attack of multiplicative noise

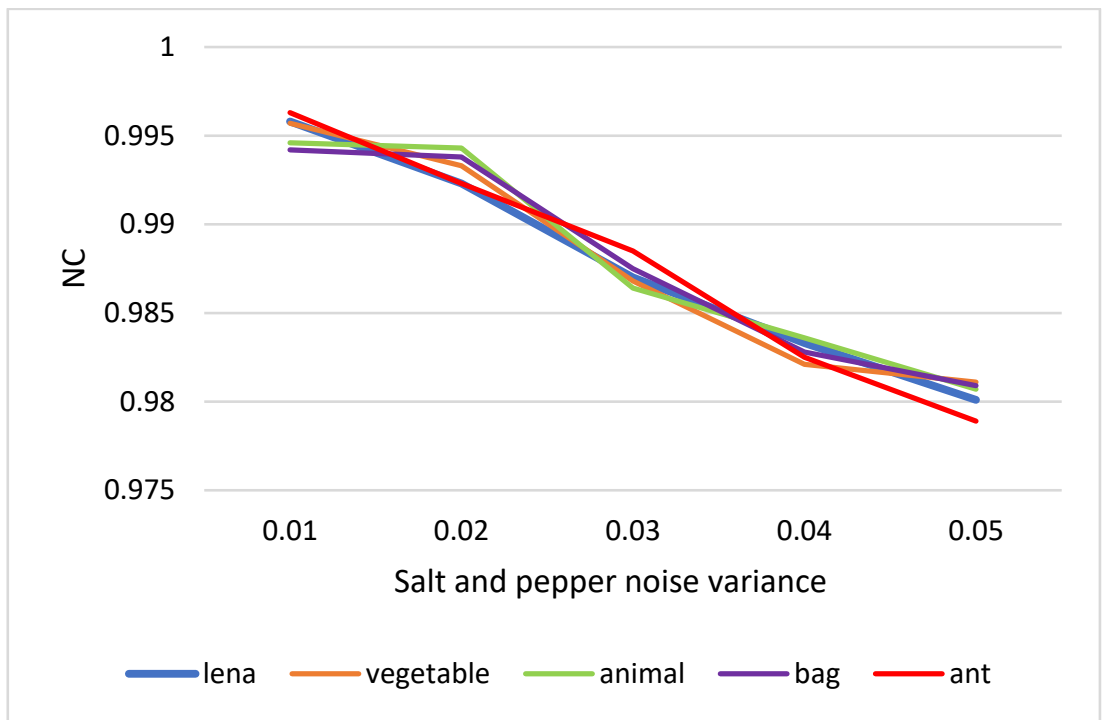

Fig. 15 Attack of salt and pepper noise 
Table 6 NC values obtained from watermark under filtering attacks

\begin{tabular}{ccccccc}
\hline Attack Types & Parameters & Lena & Vegetable & Animal & Bag & Ant \\
\hline \multirow{2}{*}{ Mean filtering } & $3 * 3$ & 0.9975 & 0.9989 & 0.9977 & 0.9993 & 0.9947 \\
& $5 * 5$ & 0.9944 & 0.9975 & 0.9965 & 0.9959 & 0.9976 \\
& $7 * 7$ & 0.9918 & 0.9960 & 0.9953 & 0.9929 & 0.9903 \\
Wiener filtering & $3 * 3$ & 0.9994 & 0.9998 & 0.9995 & 0.9997 & 0.9999 \\
& $5 * 5$ & 0.9987 & 0.9995 & 0.9988 & 0.9987 & 0.9998 \\
\multirow{2}{*}{ Median filtering } & $7 * 7$ & 0.9980 & 0.9993 & 0.9979 & 0.9978 & 0.9996 \\
& $3 * 3$ & 0.9986 & 0.9996 & 0.999 & 0.9978 & 0.9993 \\
& $5 * 5$ & 0.9966 & 0.9991 & 0.9979 & 0.9964 & 0.9983 \\
\hline
\end{tabular}

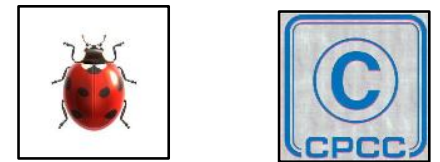

Fig. 16 Attack of sharpening and the extracted watermark

robust under noise attacks.

Moreover, in order to reflect the changes of the algorithm in different intensities under noise attacks, five kinds of salt and pepper noise and multiplicative noise attacks with different intensities are carried out. The changes of NC values under different attacks intensities are shown in Figs. 14 and 15.

According to Figs. 14 and 15, for noise attacks, the greater the noise intensity, the lower the $\mathrm{NC}$ value. This algorithm has a good robustness against noise attacks.

(2) Filtering attack

It can be seen from Fig. 16 that the watermark extracted after sharpening attack on Fig. 10e is still very clear.

As shown in Table 6, the NC values obtained from watermark under filtering attacks are all very high. The highest NC value is 0.9999 and the lowest is 0.9903, with the average value is 0.9951. Due to the combination of NSCT and SVD, the change in the low frequency coefficients of each sub-block is small under filtering attacks. The results show that the algorithm is robust under filtering attacks.

(3) Geometric attack

Common geometric attacks include cropping attacks, rotation attacks and row-column offsets. The cropping methods in this paper are 1/16 in the upper left corner and 1/32 the upper left corner. The results are shown in Fig .17 and 18 .

As shown in Figs. 17 and 18, the most of information in the upper left corner of the image is lost by cropping, and the validity of copyright watermark after extraction are less. Due to the block processing when constructing the feature matrix and singular value decomposition of each sub-block, so that proposed zero-watermarking algorithm can effectively resist local cropping attack.

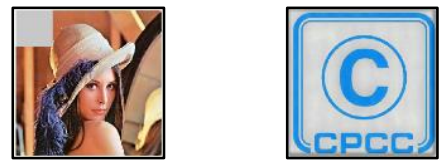

Fig. 17 Attack of 1/16 cropping in the upper left corner and the extracted watermark
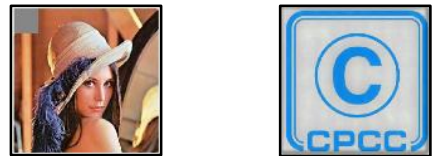

Fig. 18 Attack of 1/32 cropping in the upper left corner and the extracted watermark

\section{Discussion}

In the proposed zero-watermarking algorithm, a colour video will generate many zero-watermarks from extracted key-frames. The zero-watermarks generated by multiple key-frames can effectively resist malicious clipping of key-frames in the actual state of secondary creation, and prevent key-frames being clipped to extract effective feature matrices, so as to get the copyright watermarks. By this way, even if the key-frames are clipped, watermarks which are extracted still keep a good clarity. Besides, the propoesd zerowatermarking algorithm has two times of chunking in the generation of feature matrices. The first processing is before NSCT. When the colour video is attacked by cropping attack, the coefficients after NSCT can be independent of each other. The second processing makes the subsequent SVD more stable. In addition, the separation of RGB in this algorithm enhances security of the system, because the three zerowatermarks are required to extract a colour watermark, and the separation of RGB achieves a triple encryption logically with Arnold Transform. Above all, the fundamental feature of the zero-watermarking algorithm is that copyright protection is realized without any modification of the video. This zero-watermarking algorithm generates Key $K_{1}$, which will be kept by the owner of video copyright. Key $K_{2}$ can be uploaded to the blockchain network. In process of 
copyright certification, the user provides the Key $K_{1}$ to match the key pairs $\left(K_{1}, K_{2}\right)$ to extract copyright watermarks from zero-watermarks on the blockchain.

In the video copyright protection system, the blockchain network replaces the third-party notary in the traditional zero-watermarking scheme and avoid the security issues that exist with universal third-party notaries. Furthermore, excellent security control mechanisms exist in the blockchain itself. The system is applied in the field of video copyright protection is a scenario where data security requirements are high, so accessing control of data on the alliance chain is required, which is mainly divided into the following two aspects:

- Access control of communication data on the chain is accomplished through node certificates and SSL.

- In the access control of node storage data, the blockchain uses disk encryption, which is performed within the institution. In the institution's intranet, each institution independently encrypts the node's rigid disk data. When the rigid disk of the machine is away from the institution and the node is allowed to start up on a network outside the institution's intranet. The rigid disk data will not be decrypted and the node will not be able to start up and thus steal the data on the federated chain.

In addition, the data on the chain (the colour zero-watermark) has been scrambled. Even if being accessed, no valuable information can be obtained. Copyright rights transactions will only be done on the blockchain network without any further manipulation of the video. This way is more efficient than traditional methods. For example, all permission transactions and permission ownership information will be recorded on the blockchain network and be protected from malicious tampering. Thus, the malicious user uses and reproduces a video illegally, a legally valid proof will be found through fast traceability in the video copyright protection system.

\section{Conclusion and future work}

Aiming at the problems of the traditional watermarking scheme for video copyright, such as poor robustness, low security, weak invisibility and third-party mechanism, we propose a zerowatermarking protection scheme for video copyright based on blockchain and NSCT + SVD.

In our scheme, firstly, the proposed key-frames extraction method performs high accuracy in filtering key-frames. Secondly, we choose the NSCT which is combined with the Nonsubsampled Pyramid (NSP) and the
Nonsubsampled Directional Filter Bank (NSDFB) in zero-watermarking algorithm. This method can decompose the images in multi-scale and multi-direction, making up for the deficiency that wavelet transform only has three directions of vertical, horizontal and diagonal transformation of the image. It has good translation invariance, making up for the shortcomings of contourlet transform sampling. Thirdly, video zero-watermarking algorithm based on SVD can achieve the stability and video quality will not have any impact. Fourthly, the difficulties in security and efficiency of video copyright protection are overcome by combining blockchain and the video zero-watermarking algorithm.

Further works are as follows:

- Investigate a copyright protection method about the secondary creation registration problem with blockchain and digital watermarking technology at a low cost.

- Investigate an algorithm improving the reliability of the watermark and the efficiency of block for video copyright protection system.

- Study cross-chain key technologies so as to realize protection of cross-domain video copyright.

\section{Acknowledgments}

We sincerely thank the Reviewers and the Editor for their valuable suggestions.

\section{Authors' contributions}

Xiangqi $\mathrm{Wu}$ and Peng $\mathrm{Ma}$ designed the framework of this scheme. Zihan Jin and Yuxuan $\mathrm{Wu}$ designed the core algorithm and performed the experiments. Wei Ou provided technical supports. All authors reviewed and approved final manuscript.

\section{Authors' information}

Xiangqi $\mathbf{W u}$ is a student in Hainan University. His research interests include cyber security and blockchain technology.

Peng $\mathrm{Ma}$ is a student in Hainan University. His research interests include information security and artificial intelligence.

Zihan Jin is a student in Hainan University. His research interests include federal learning and blockchain technology. Yuxuan $\mathbf{W u}$ is a student in Hainan University. His research interests include cryptography and blockchain technology.

Wei Ou received the B.S. degree from the Air Force Engineering University, in 2000, and the M.S. degree in the National University of Defense Technology in 2005, and the Ph.D. degree in the National University of Defense Technology, in 2013. He is an associate professor in Hainan University. His research interests include cryptography, cyber security, Al security and Blockchain technology.

Wenbao Han received his M.S. degree at Sichuan University in 1988 and the Ph.D. degree at Sichuan University in 1994. $\mathrm{He}$ is currently a professor in Hainan University. His research interests include cryptography, cyber security, Al security and Brain-like computing.

Funding

Not applicable. 
Availability of data and materials

There is no supporting data available.

\section{Competing Interests}

The authors declare that they have no competing interests.

\section{References}

1. H. Gao, C. Liu, Y. Li and X. Yang (2020) V2VR: Reliable hybrid-network-oriented $\mathrm{V} 2 \mathrm{~V}$ data transmission and routing considering RSUs and connectivity probability. IEEE Transactions on Intelligent Transportation Systems. doi: 10.1109/TITS.2020.2983835.

2. Xia W (2017) Research status and development of digital watermarking. The Guide of Science \& Education 2017(3):161-161.

3. Zhao Q (2004) Digital watermarking copyright protection system. Beijing: Capital University of Economics and Trade.

4. Guo X (2020) Block chain technology rebuilds the new order of media industry -- take baidu totem as an example. Science and Technology Communication 12(9): 4-6+24.

5. Ruan X (2020) Research on financial problems of supply chain finance model embedded based on block chain technology -- a case study of ant financial double-chain communication. Shanxi Agricultural Economics 10:162-163.

6. Atsushi, KOBAYASHI, Akira, HASEGAWA, Miho, FUKUDA (2017) A practice of copyright protection applying digital watermark technology: studying applications in still pictures and moving pictures, and exploration of future expectations. Journal of Information Processing \& Management. doi:10.1241/johokanri.60.89.

7. He F (2020) Digital watermarking and its anticompression robustness test based on wavelet domain. Automation and Information Engineering 44(5): 42-44+50

8. Zai R, WANG, Babak, SHIRI, Dumitru, BALEANU (2020) Discrete fractional watermark technique. Frontiers of Information Technology \& Electronic Engineering 6: 880-883.

9. Li Y, Wei D, Zhang L (2020) Double-encrypted watermarking algorithm based on cosine transform and fractional fourier transform in invariant wavelet domain - sciencedirect. Information Sciences.

10. Wen Q, Sun T, Wang S (2003) Concept and application of zero-watermarking. Journal of Electronics 2: 214216.

11. Wang $C$ et al (2018) Stereo image zero-watermarking algorithm based on ternary polar harmonic fourier moments and chaotic mapping. Information Sciences 450:141-156

12. Xue H, Chi W, Liu X (2020) A zero-watermarking algorithm based on NSST and Hessenberg decomposition. Modern Computer 10:89-93+103.

13. Amiri, Ali, and S. Mirzakuchaki (2020) A digital watermarking method based on NSCT transform and hybrid evolutionary algorithms with neural networks. SN Applied Sciences 2(10):1-15.

14. Xuan M (2011) H. 264/AVC video integrity authentication key technology research. Hefei University of Technology.

15. Meng H (2020) Research on video encryption technology based on H. 264 coding standard. Northeast University of China.

16. Liu X (2020) An overview of video digital watermarking technology. Television Technology 44(5):11-15.

17. Sun $Y$ et al (2020) Research on scalable video watermarking algorithm based on H. 264 compressed domain. Optik.

18. Qiu Y, Xue F, Tang J (2019) Spatio-temporal feature analysis combined with random key for compressed domain digital video watermark embedding and extraction method. Computer Applications Researchvol 36(9):2813-2817.

19. Guo Z, Zhang L, Zhang D (2010) A completed modeling of local binary pattern operator for texture classification. IEEE Transactions on Image Processing 19(6):1657-1663.

20. Ojala, T, M. Pietikainen, T. Maenpaa (2002) Multiresolution gray-scale and rotation invariant texture classification with local binary patterns. IEEE Transactions on Pattern Analysis \& Machine Intelligence 24(7):971-987.

21. Q Kou et al (2019) Texture target classification integrating CLBP and local geometric features. Photoelectric engineering 46(11):66-73.

22. Xu S et al (2013) Texture descriptors for spatial statistical features based on LBP values. Pattern Recognition and Artificial Intelligence 26(8):769-776.

23. Yang $J$ et al (2016) Research on undersampling methods based on data density distribution. Computer Applications Research 33(10):2997-3000.

24. Liu J, Zhao W, Zhu H (2014) Pattern recognition: pattern recognition. Harbin Institute of Technology Press.

25. Deng S (2015) Robust watermarking algorithm for wavelet transform and singular value decomposition. Laser Journal 36(4):86-89.

26. Guo L et al (2019) Fingerprint watermarking authentication algorithm based on SM2 and singular value decomposition (SVD). Journal of Beijing Institute of Printing 27(2):46-50.

27. Hao X, Peng G (2017) Video summarization based on SVD and sparse subspace clustering. Journal of Computer-Aided Design and Graphics 29(3):485-492.

28. Xiao $Z$ et al (2017) Enhanced singular value decomposition and cellular neural networks for zerowatermarking. Chinese Journal of Graphical Graphics 3:1144 - 1148+1153

29. Wang L et al (2018) Digital watermarking algorithm in wavelet domain based on Arnold scrambling and logistic chaotic encryption. Information Technology 49 $-53+58$

30. Chen Y (2019) Research on colour image encryption algorithm based on chaotic random chunking and Arnold transform. Digital Technology and Applications 37(6):135-137.

31. Wu L, Zhang J et al (2010) Arnold transform and its inverse transform. Microcomputer Information 26(14):206-208

32. Guo $L$ et al (2019) Fingerprint watermarking authentication algorithm based on SM2 and singular value decomposition (SVD). Journal of Beijing Institute of Printing 27(2):46-50. 


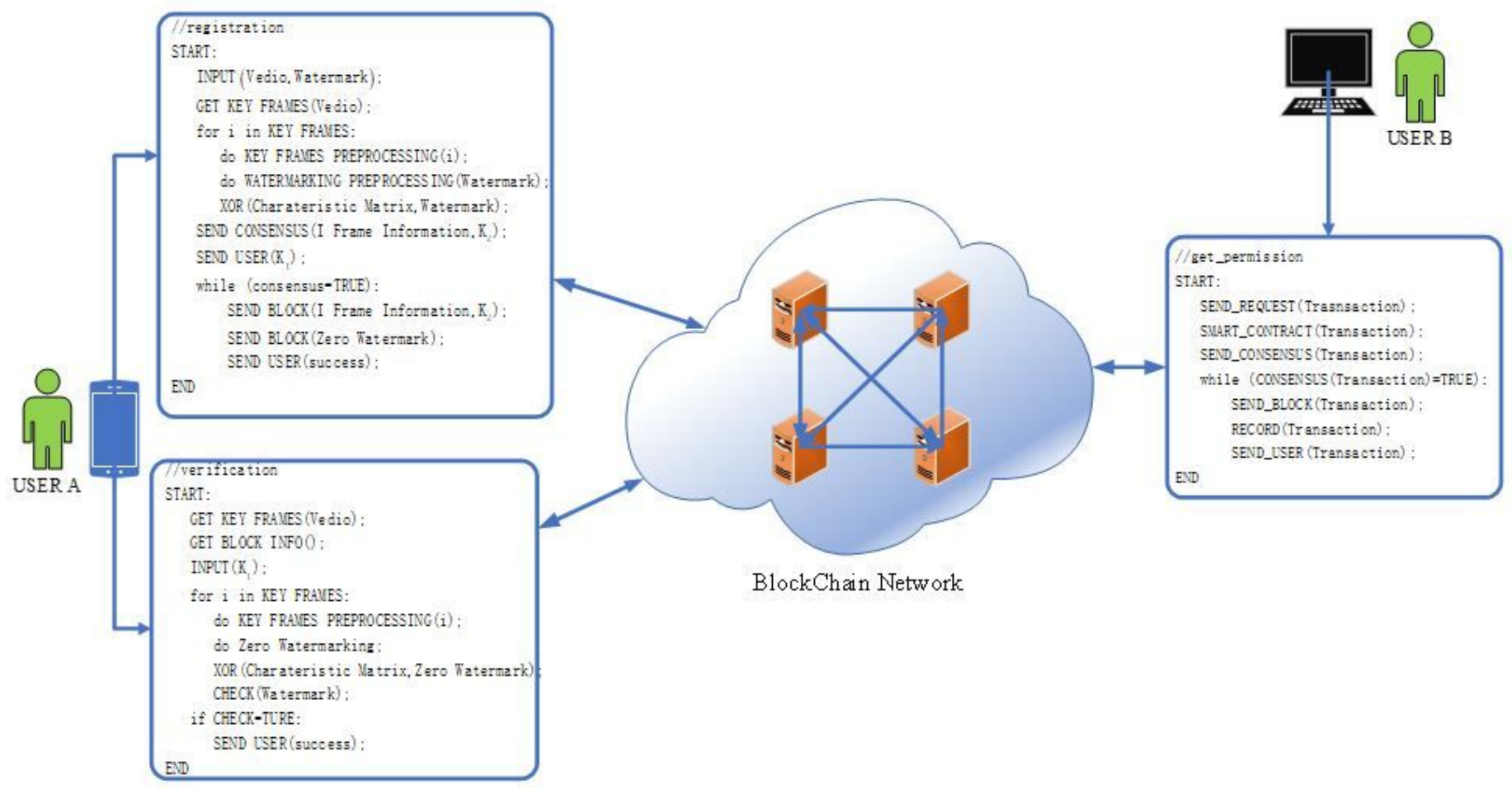

Figure 1

Framework of the protection system 


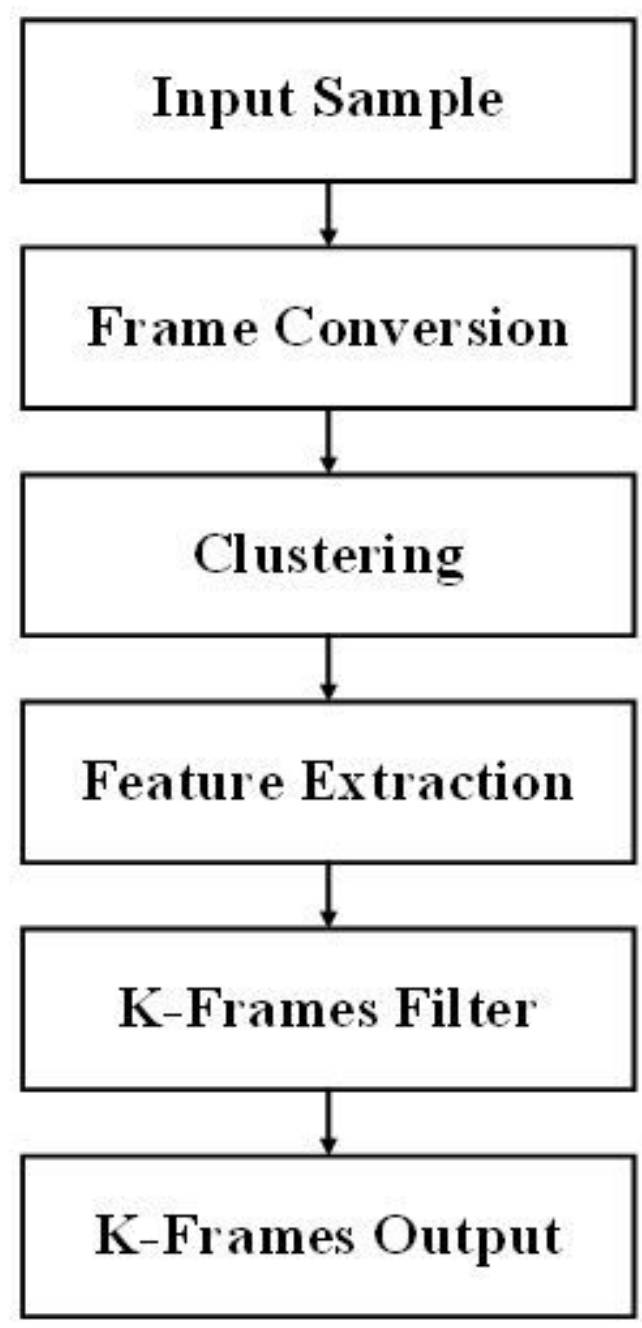

Figure 2

Process of key-frames extraction

\begin{tabular}{|c|c|c|}
\hline 9 & 12 & 34 \\
\hline 10 & 25 & 28 \\
\hline 99 & 64 & 56 \\
\hline
\end{tabular}

(a) $3 p x^{*} 3$ pximage

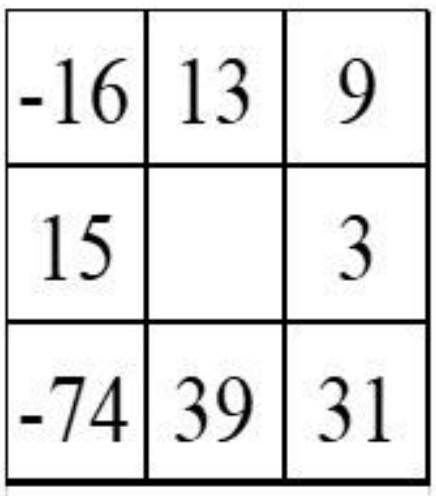

(b) Local Difference Images

Figure 3 


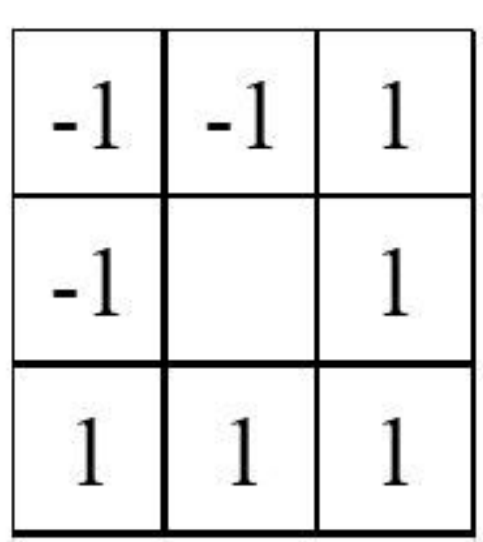

(a) CLBP_S

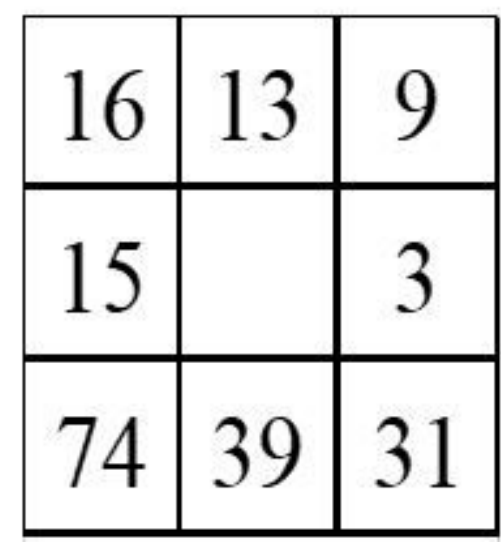

(b) CLBP_M

Figure 4

Description of CLBP algorithm II

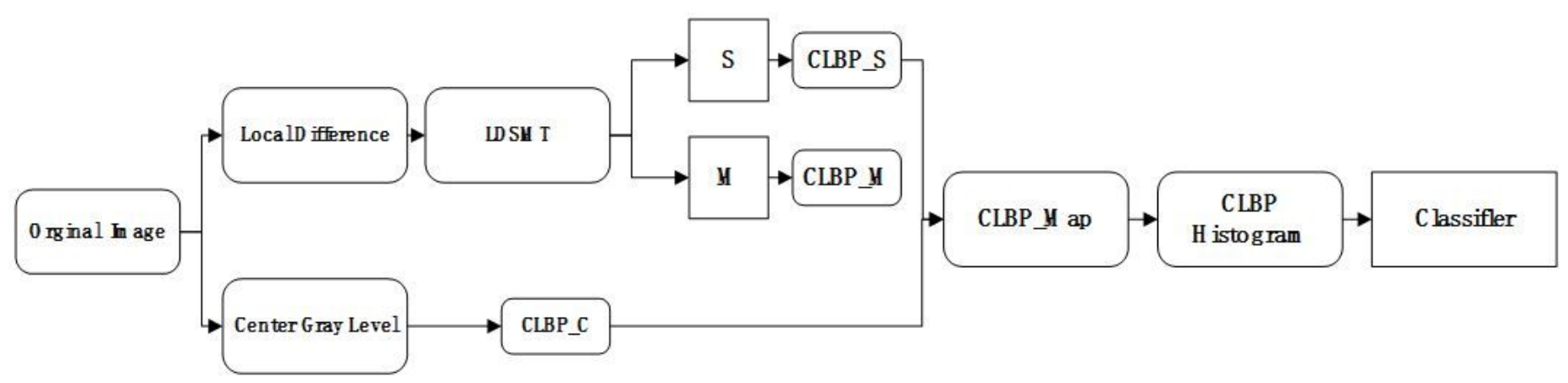

Figure 5

CLBP algorithm 


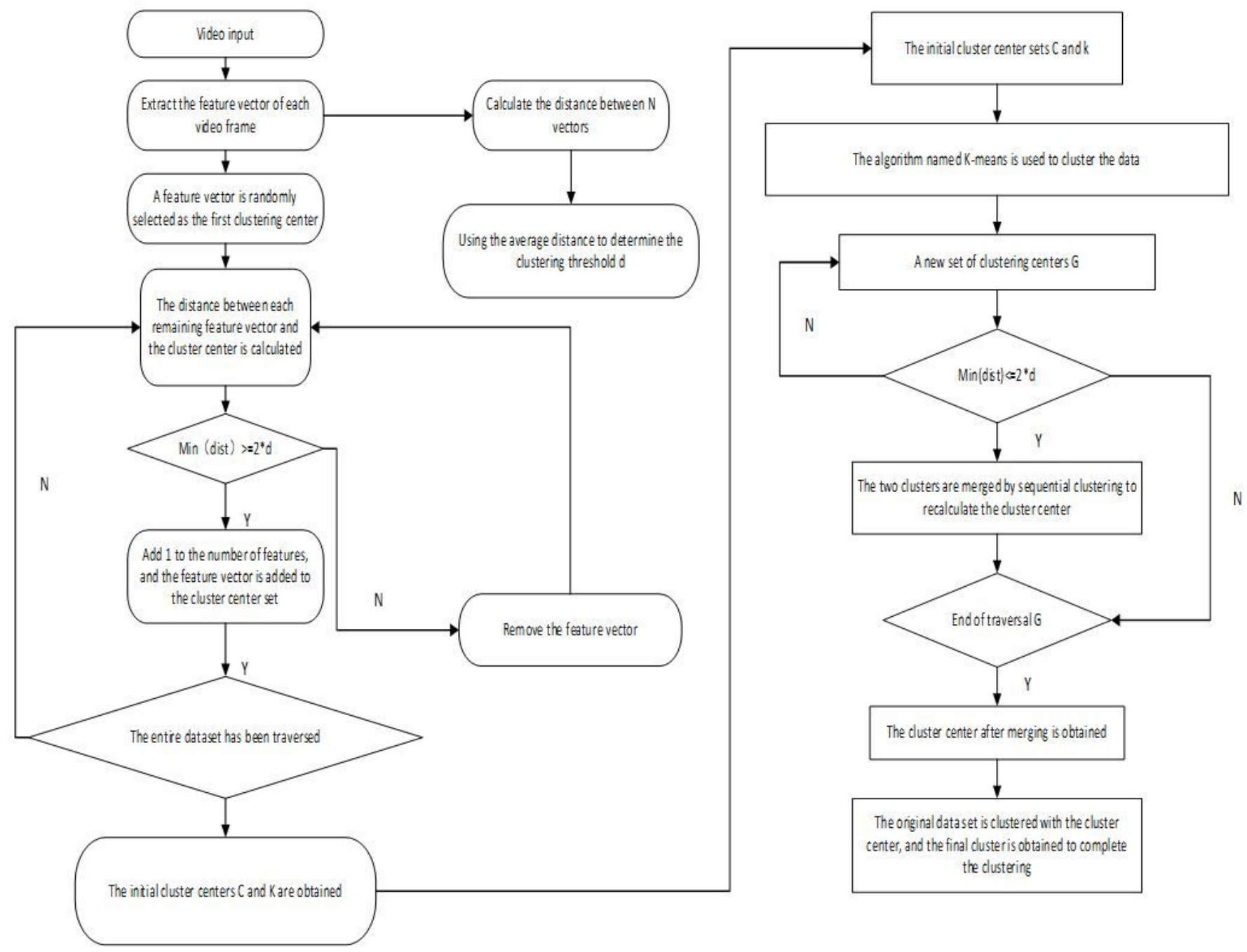

Figure 6

Process of clustering algorithm 


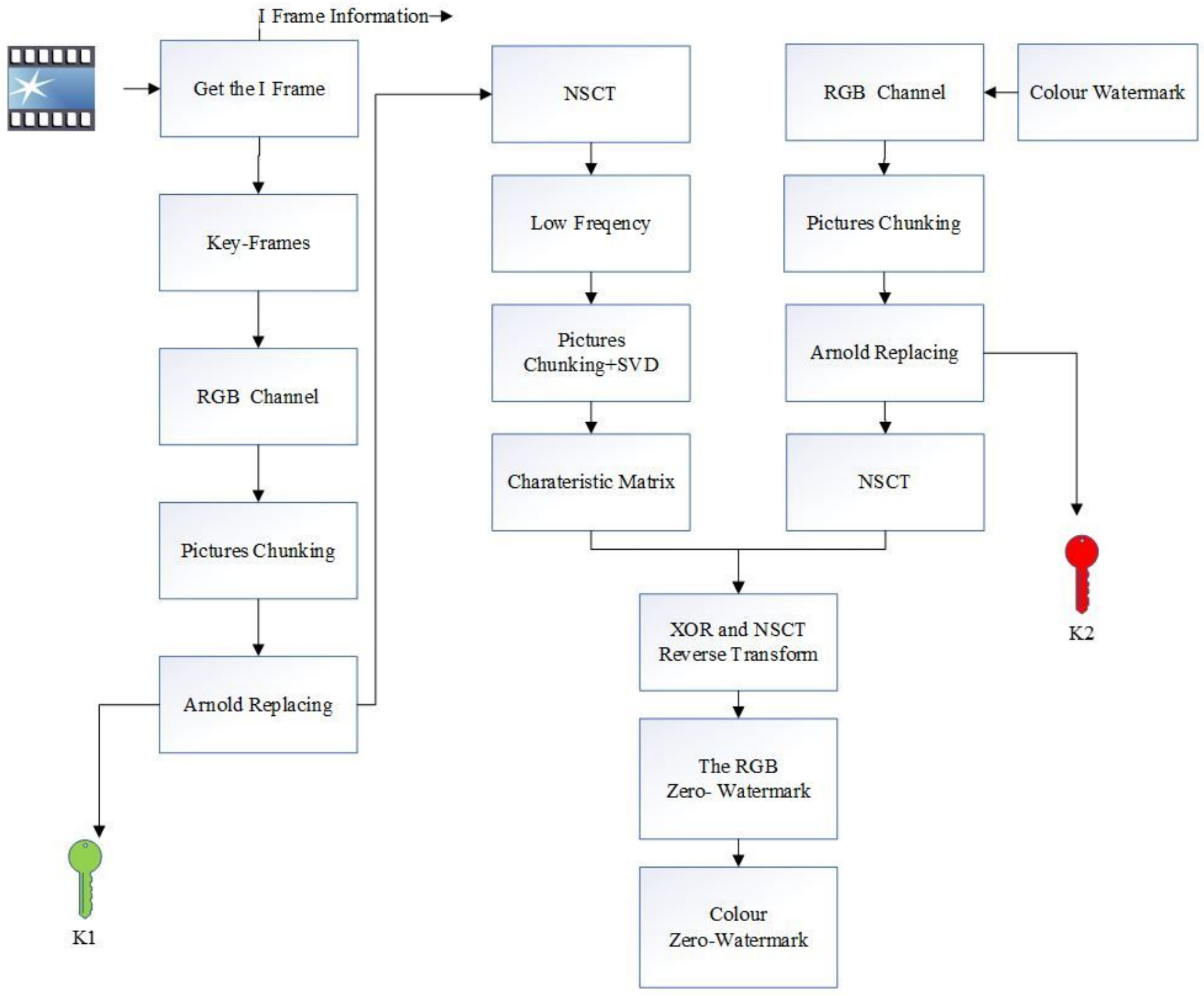

Figure 7

Process of watermark generation 


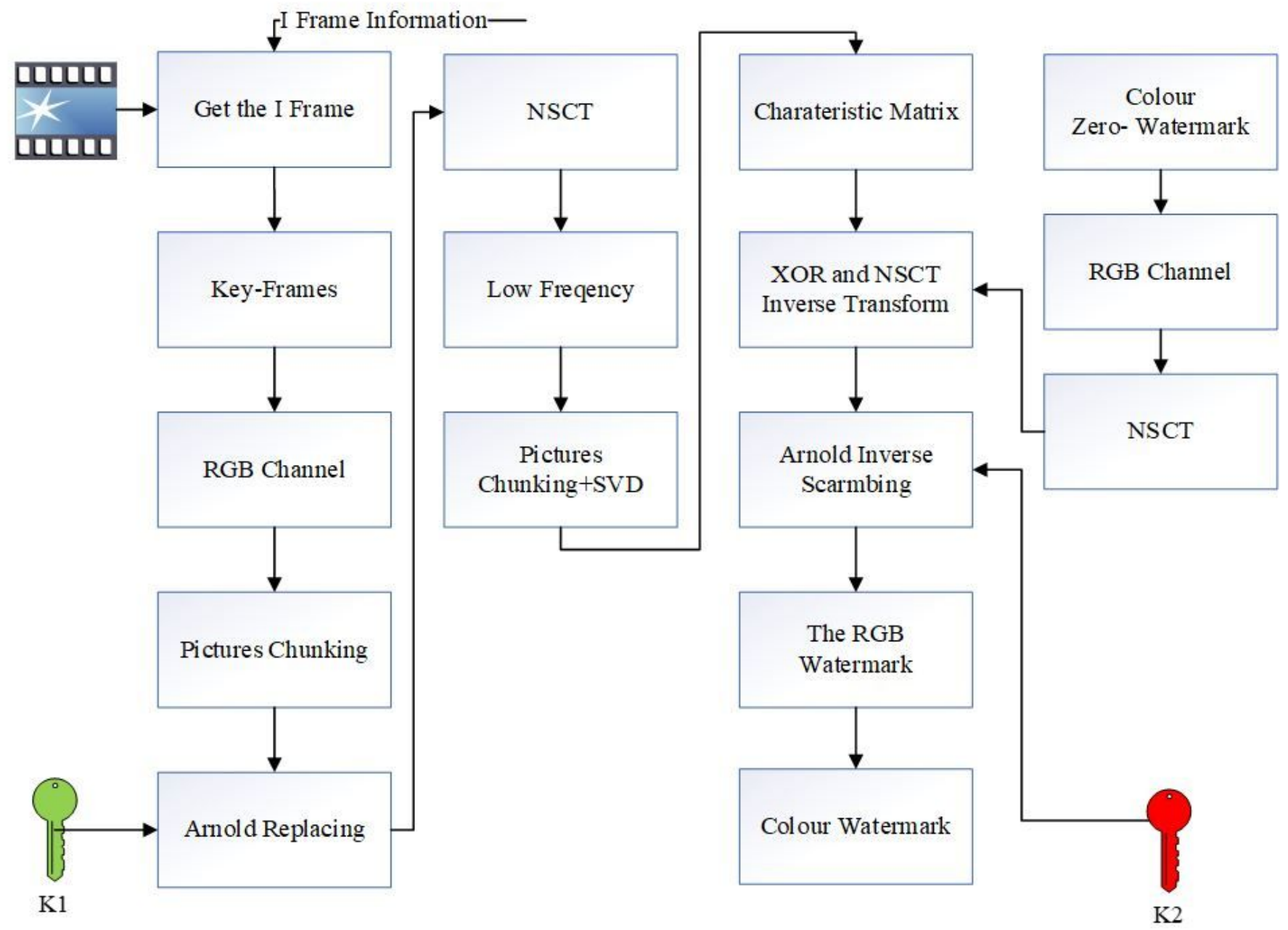

Figure 8

Process of watermark extraction 


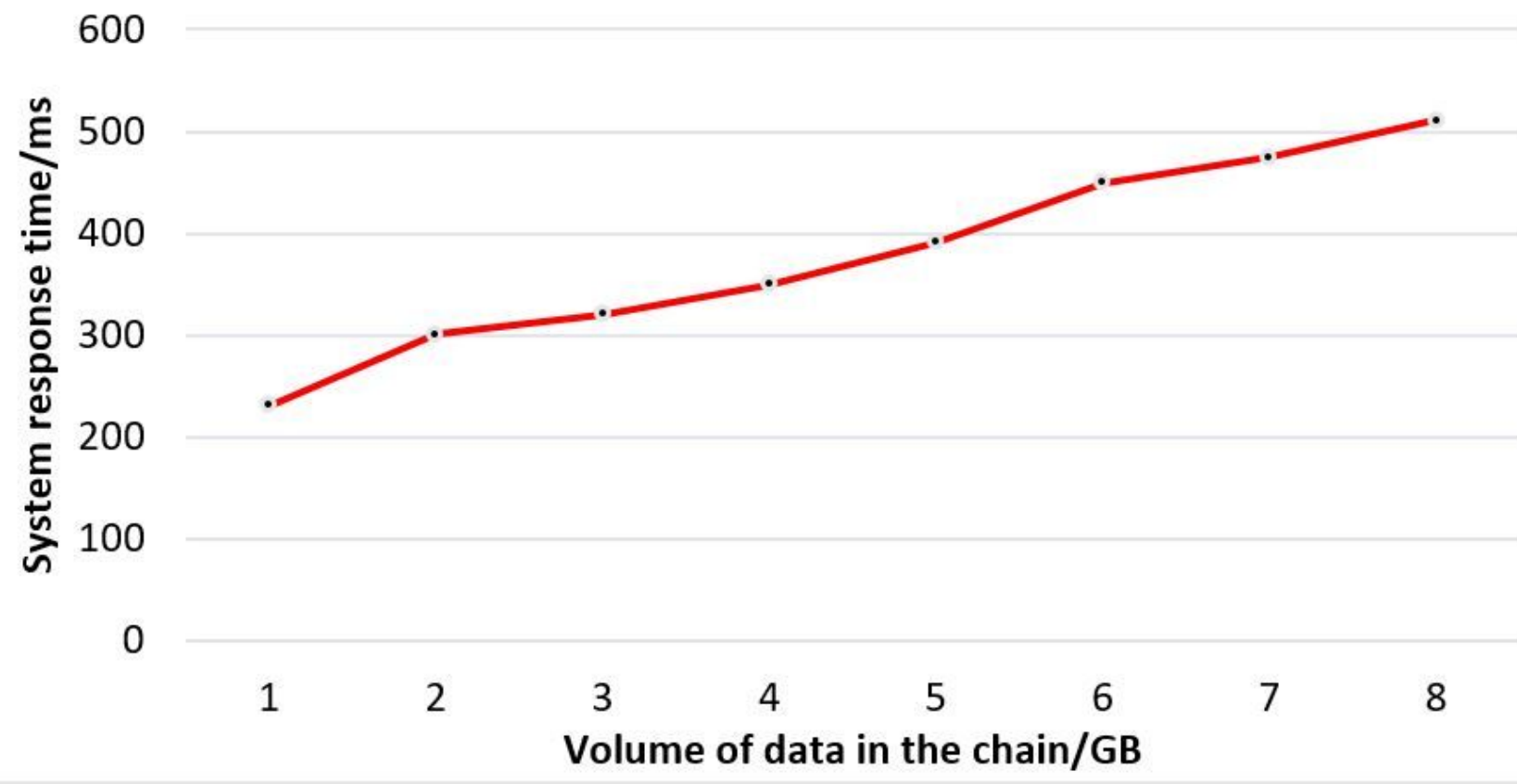

Figure 9

System response time and on-chain data volume testing 


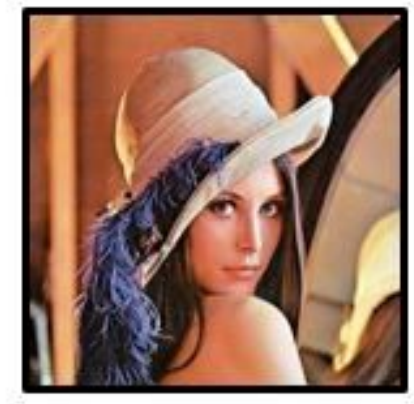

(a)len

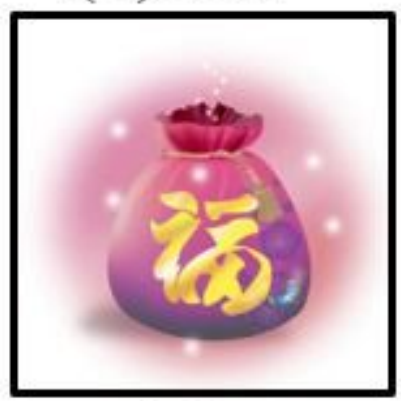

(d)bag

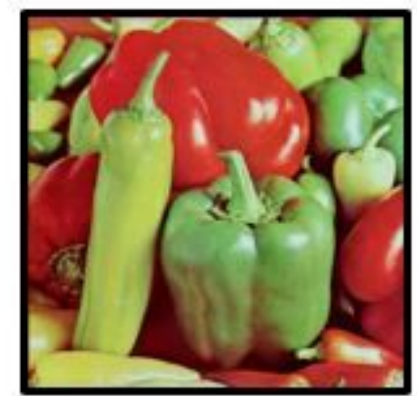

(b)vegetable

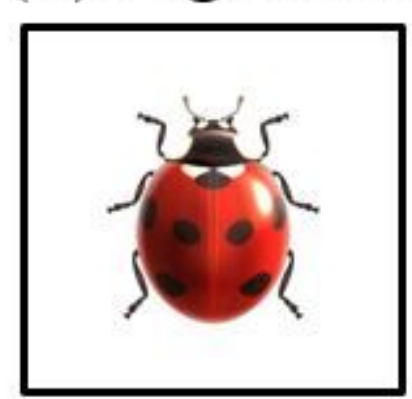

(e)ant

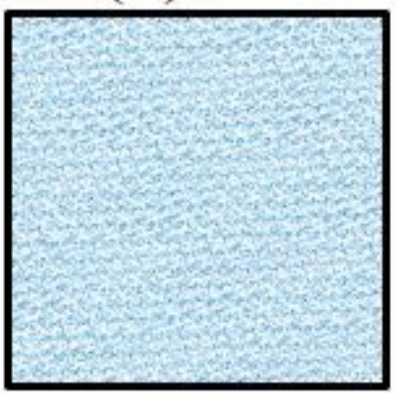

(g)zero-watermark

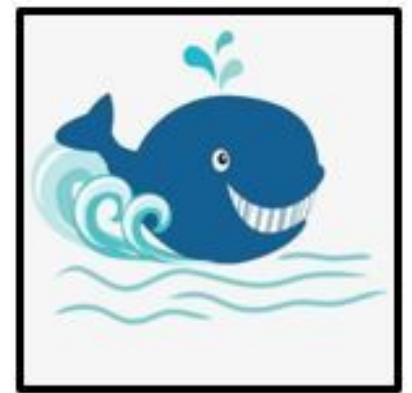

(c)animal

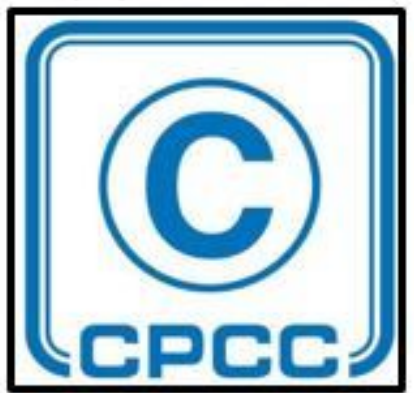

(f)CPCC

Figure 10

Images, copyright watermarking and zero-watermark
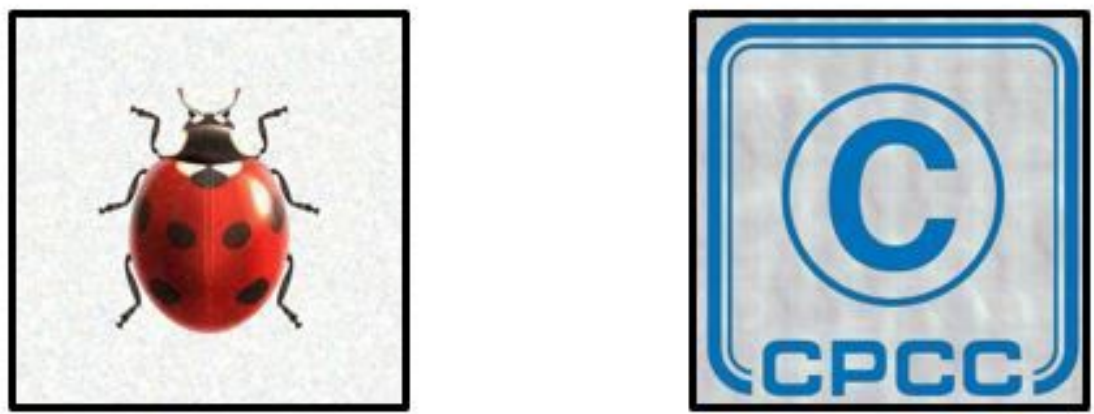

Figure 11 

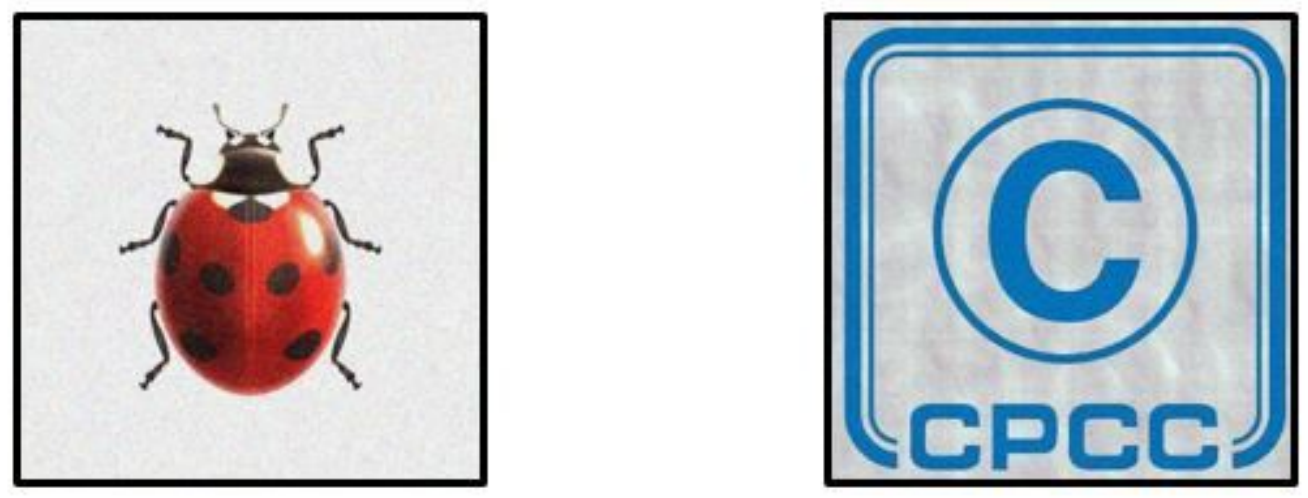

Figure 12

Attack of gaussian noise (0.03) and the extracted watermark
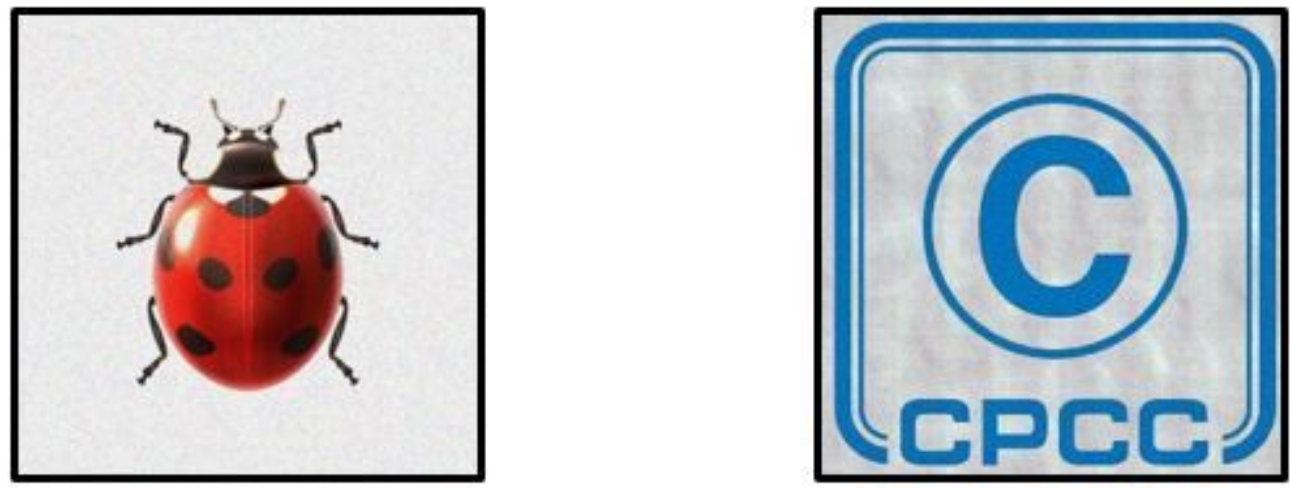

Figure 13

Attack of multiplicative noise (0.03) and the extracted watermark 


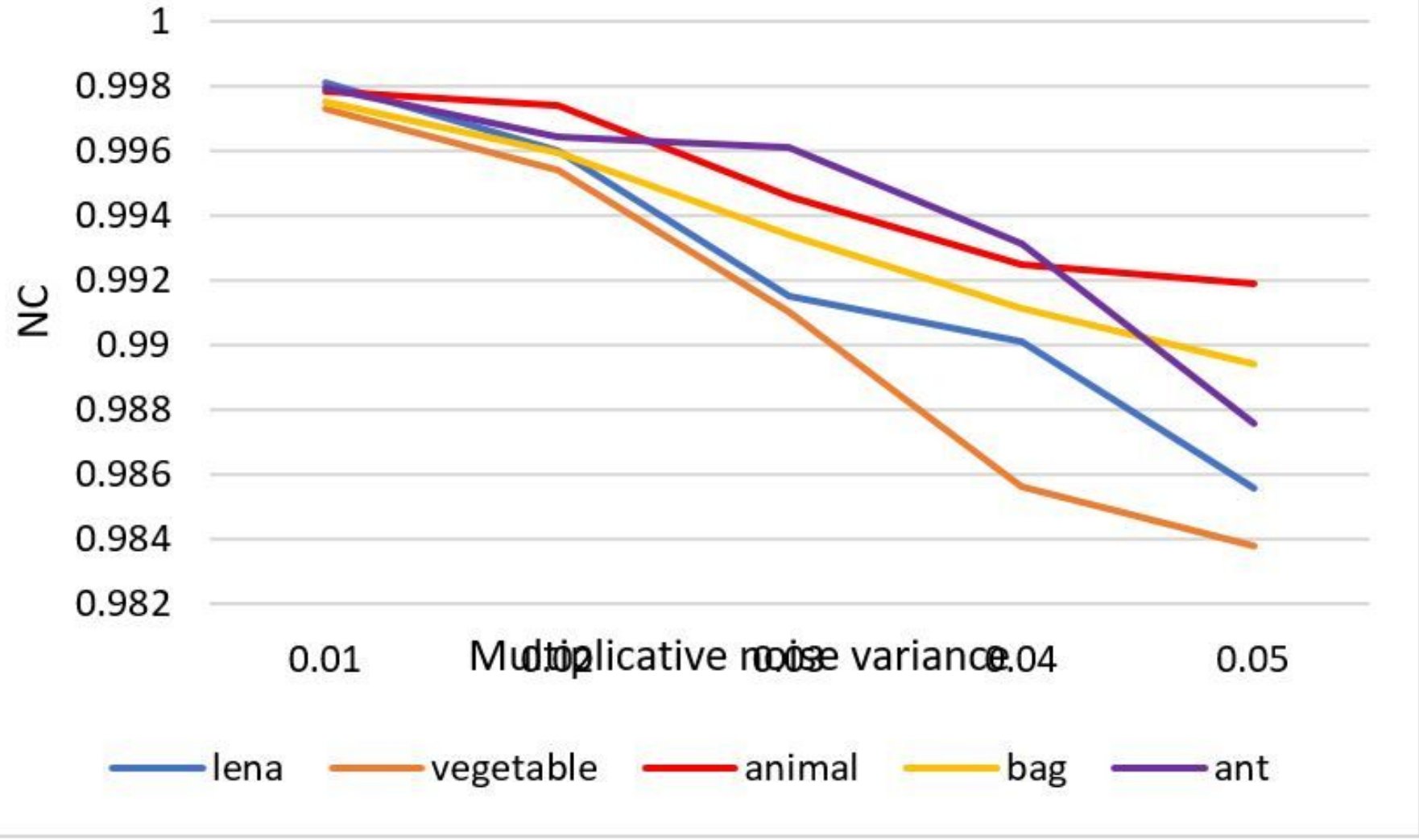

Figure 14

Attack of multiplicative noise 


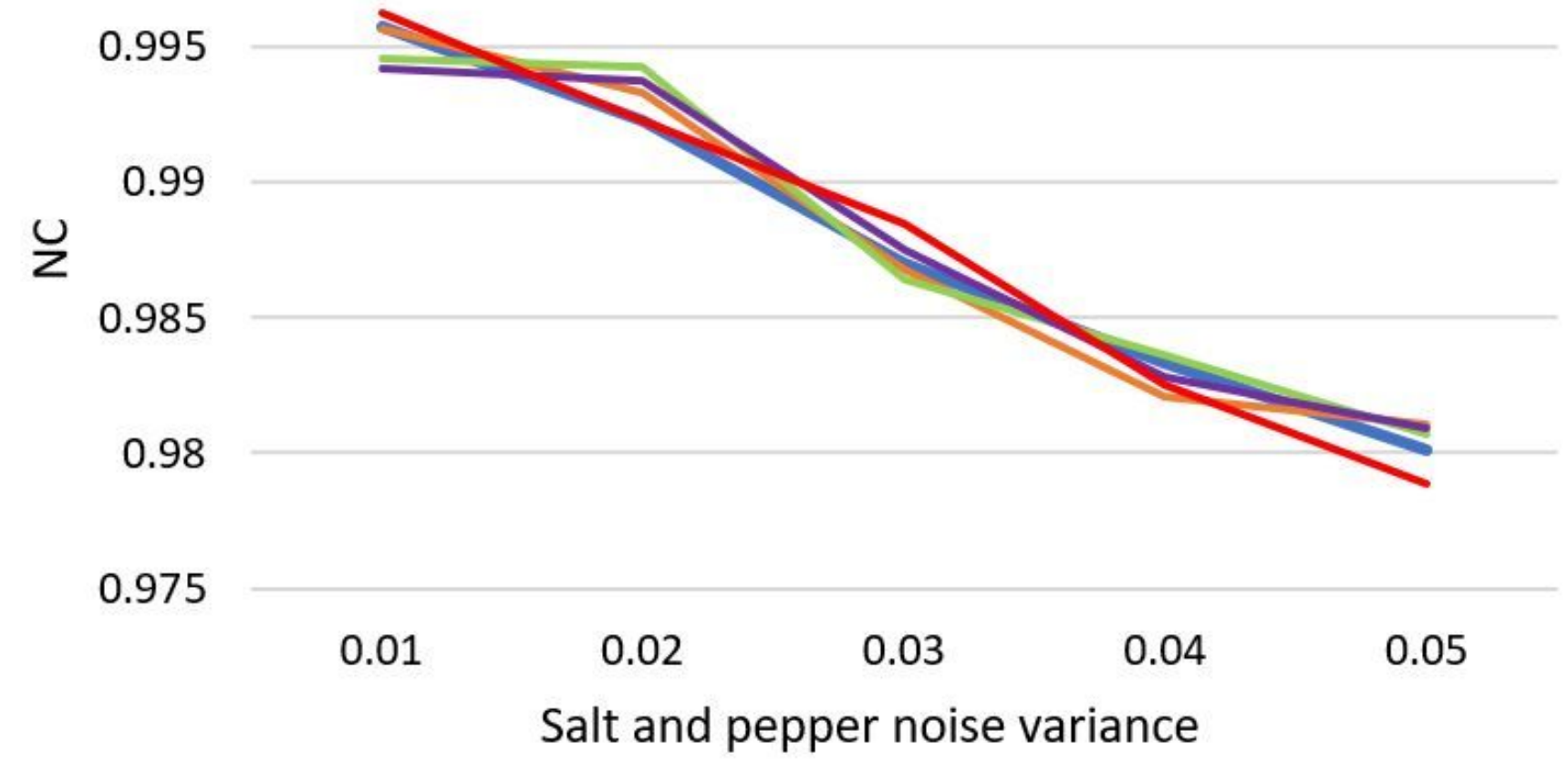

lena vegetable $\longrightarrow$ animal bag $\longrightarrow$ ant

Figure 15

Attack of salt and pepper noise
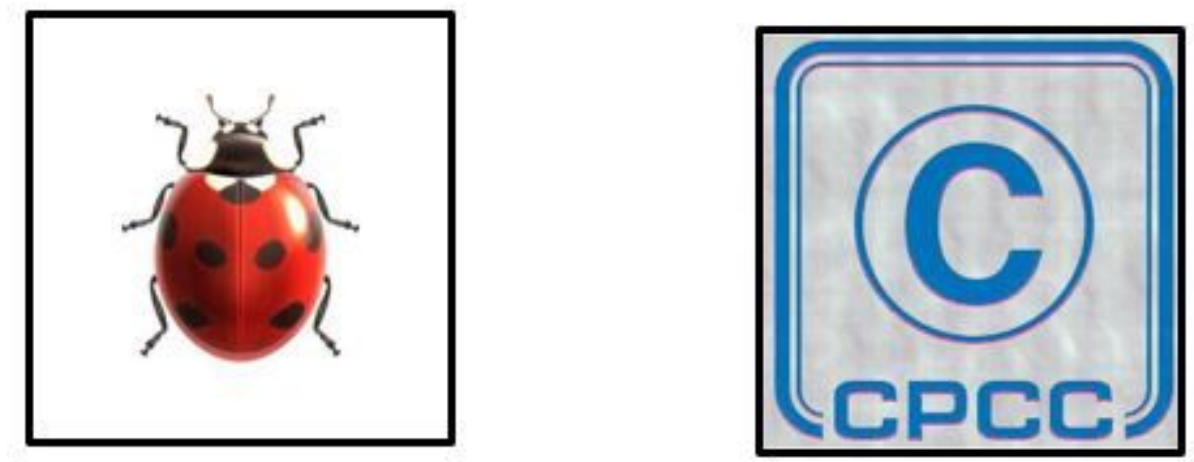

Figure 16

Attack of sharpening and the extracted watermark 

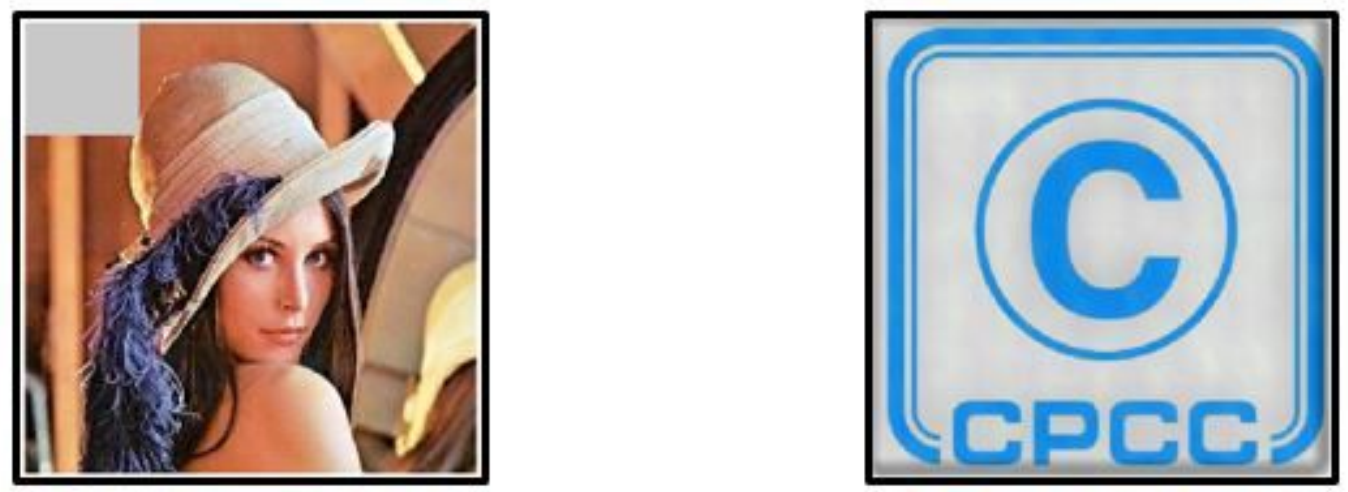

Figure 17

Attack of 1/16 cropping in the upper left corner and the extracted watermark
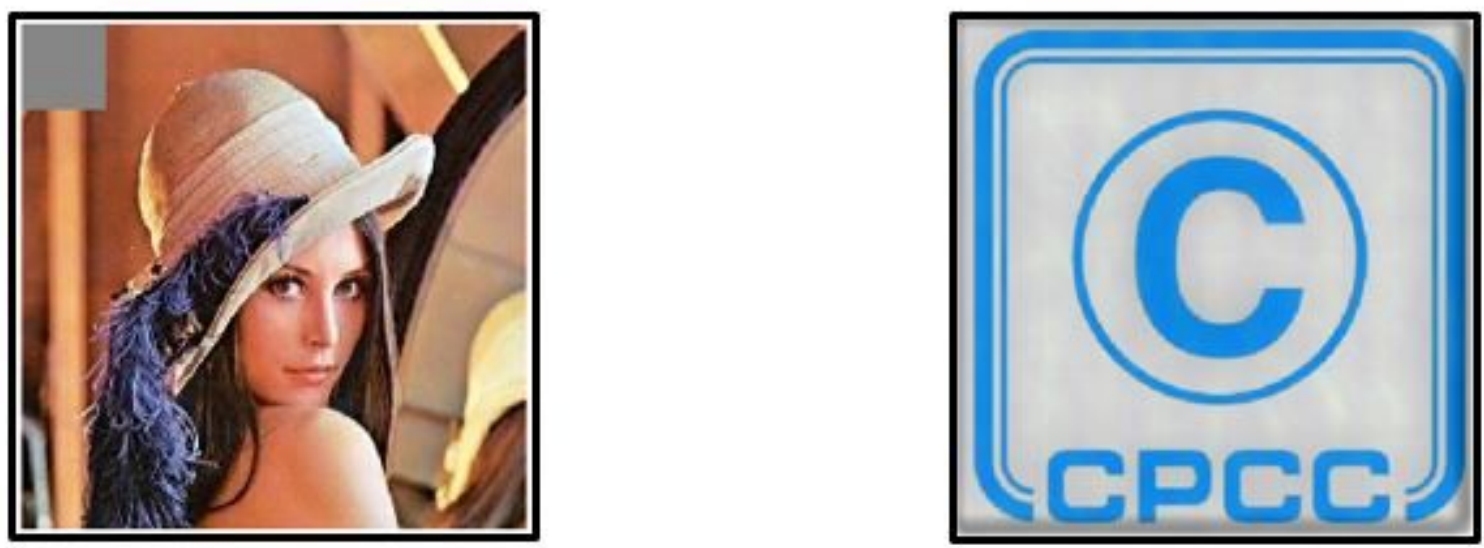

Figure 18

Attack of $1 / 32$ cropping in the upper left corner and the extracted watermark 